\title{
Search for Charm
}

\author{
MARY.K. GAILLARD * and BENJAMIN W. LEE \\ Fermi National Accelerator Laboratory, Batavia, Illinois 60510 \\ and \\ $\checkmark$ JONATHAN L. ROSNER \\ University of Minnesota, Minneapolis, Minnesota 55455
}

\section{ABSTRACT}

A systematic discussion of the phenomenology of charmed particles is presented with an eye to experimental searches for these states. We begin with an attempt to clarify the theor etical framework for charm. We then, discuss the SU(4) spectroscopy of the lowest lying baryon and meson states, their masses, decay modes, lifetimes and various production mechanisms. We also present a brief discussion of searches for short-lived tracks. Our discussion is largely based on intuition gained from the familiar -- but not necessarily understood -- phenomenology of known hadrons, and predictions must be interpreted only as guidelines for experimenters.

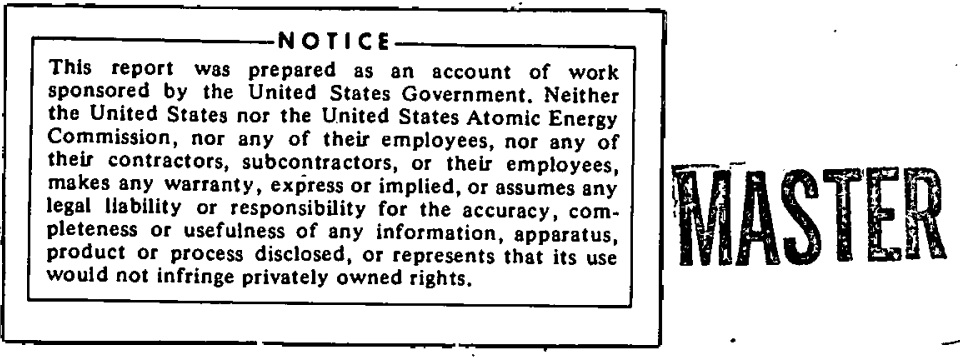

* On leave of absence from Laboratoire de Physique' Theorique et Particules Elementaires, Orsäy (Laboratoire associé au CNRS). 


\section{DISCLAIMER}

This report was prepared as an account of work sponsored by an agency of the United States Government. Neither the United States Government nor any agency Thereof, nor any of their employees, makes any warranty, express or implied, or assumes any legal liability or responsibility for the accuracy, completeness, or usefulness of any information, apparatus, product, or process disclosed, or represents that its use would not infringe privately owned rights. Reference herein to any specific commercial product, process, or service by trade name, trademark, manufacturer, or otherwise does not necessarily constitute or imply its endorsement, recommendation, or favoring by the United States Government or any agency thereof. The views and opinions of authors expressed herein do not necessarily state or reflect those of the United States Government or any agency thereof. 


\section{DISCLAIMER}

Portions of this document may be illegible in electronic image products. Images are produced from the best available original document. 
TABLE OF CONTENTS

1. Prologue

2. Spectroscopy

$\begin{array}{ll}\text { Quarks } & \stackrel{4}{4} \\ \text { Baryons } & 20 \\ \text { Mesons } & \stackrel{15}{\sim}\end{array}$

3. Masses of Charmed Particles

3. 1 Nature of SU(4) Symmetry Breaking

3.2 Pseudoscalars

3. 3 Vector Mesons

3. 4 Spin $1 / 2$ Baryons

4. Decays of Charmed Particles

4. 1 Mesons -- Leptonic Decays'
4.1.1 Two-body Decays
4.1.2. Three-body Decays
4.1.3 Multi-pion Decays

4.1.4 Inclusive Semileptonic Estimate

4. 2 Mesons -- Nonleptonic Decays

4. 2.1 Quark Model

4.2.2 A Specific Two-body Final State

4. 2. 3 Statistical Model

4.2.4 $D^{\circ}-\bar{D}^{\circ}$ Mixing
4.2. 5. Mass Spectra and Exotic Combinations.
4. 3 Baryon Decays
4. 4 Vector Meson Decays

5. Production of Charmed Particles

5. 1 Diffractive Production of $\phi_{c}$

5. 2 Neutrino Reactions

5. 2. 1 Quasi-elastic Scattering

5.2.2 Diffractive Production of Vector Mesons

5. 2.3. Deep Inelastic Production

5.3 è Annihilation

'5. 4 Lepton Production at High Momentum Transfer

5. 5 Associated Production in Strong Interactions

6. Detection of Tracks of Charmed Particles

7. Summary and Conclusions 


\section{PROLOGUE}

Both theoretical developments ${ }^{1}$ in the study of spontaneously broken gauge theories and the experimental observation ${ }^{2}$ of neutral currents point in the direction of a unified, renormalizable theory of weak interactions. However, other ingredients are necessary for the successful realization of such a theory; one possibility involves a fourth "charmed" quark, ${ }^{3}$ implying the existence of a new spectrum of hadron states.

Let us review the current status of the theoretical background on charmed particles. In order to present conflicting views (which exist even among ourselves), we shall utilize a fictitious dialogue between two researchers -- an enthusiast and a devil's advocate.

A: So if one adopts the view that the Weinberg-Salam, model ${ }^{4}$ is essentially correct, a viewpoint consonant with the observations of neutral current effects at various laboratories ${ }^{2}$, then one seems to be driven to the conclusion that some new degrees of freedom--new fields-must be present in the theory, in order to accommodate the absence of strangeness-changing neutral current. I understand that a four-quark scheme will do. Please explain this to me.

B: Forget about the strong interactions for the moment, and consider weak and electromagnetic interactions as manifestations of a single "weak" force. Then all fields are characterized by weak isospin and weak hypercharge. The world consists of four left-handed isodoublets :

$$
\left(\begin{array}{c}
\nu_{\mu} \\
\mu^{-}
\end{array}\right)_{L},\left(\begin{array}{c}
\nu_{e} \\
\mathrm{e}^{-}
\end{array}\right)_{L},\left(\begin{array}{l}
u \\
d^{-}
\end{array}\right)_{L},\left(\begin{array}{l}
c \\
s^{-}
\end{array}\right)_{L}
$$

and the right handed fields are isosinglets. Leptons and hadrons are distinguished by their weak hypercharge. When Higgs couplings are turned off, all these fields are massless and couple to massless vector bosons: a triplet which couples to weak isospin and a singlet which couples to weak hypercharge. Don't you agree that this picture is more appealing than the old one with only one quark isodoublet $\left(u, d^{\prime}\right)$ and one leftover guark $\left(\mathrm{s}^{\prime}\right)$ which doesn't couple at all to charged vector bosons? A: Perhaps. But you're not talking about the real world yet. Once you put in Higgs couplings and strong interactions, you break all that symmetry anyway. Don't you have to arrange things in an artificial way to get strangeness changing charged currents but not neutral ones? B: Notreally, if you accept the conservation of electric charge as fundamental. Strangeness changing couplings arise because, the states with well-defined masses are not the eigenstates of the weak interactions. Masses arise from Higgs couplings which can also mix fields with the same electric charge: $c \leftrightarrow$ u, s $\leftrightarrow$ d. There are really two Cabibbo angles; but one of them is not observable, since nothing changes. if we mix $c, u$ and $s, d$ by the same amount. By convention, we speak of $s, d$ 


\section{-4- FERMILAB-Pub-74/86-THŸ}

mixing. Neutral currents are diagonal in the fields $u, d, c$ and s. Since $d^{\prime}$ and $s^{\prime}$ have the same weak quantum numbers, they always appear in neutral currents in the combination $\bar{s}^{\prime} s^{\prime}+\bar{d}^{\prime} d^{\prime}$, which is invariant under the Cabibbo rotation.

A: But why should the Higgs mesons pick on quarks? Why don't they $\operatorname{mix} \mathrm{e}$ and $\mu$ ?

B: They might, but you'll never know it, as long as the neutrinos remain massless. By definition, $v_{\mathrm{e}}$ is the neutrino that couples to the physical electron.

A: What do the strong interactions do? Are you really led to an $\mathrm{SU}(4)$ symmetry?

B: Renormalization of the weak interactions requires that the strong . interactions be invariant under the weak gauge group $\left(u^{\prime} \rightarrow d^{\prime}, c \rightarrow s^{\prime}\right)$. It turns out that they are also invariant under strong isospin $(u \rightarrow d)$. Putting all these symmetries. together, you are led to SU(4) invariant couplings. SU(4) is, of course, broken by the masses.

A: Nevertheless, your lepton-hadron symmetry is broken by strong interactions. And what about color?

B: Perhaps color should be regarded as an extra degree of freedom for quarks which allows them to couple to color gauge bosons, giving rise to strong interactions. An overall symmetry may emerge in a larger scheme; Georgi and Glashow (1974) have recently discovered that the weak gauge group, together with color, can be accommodated in an
.

$$
\text { 1. } \quad \text {-5- FERMILAB-Pub-74/86-THY }
$$

SU(5) gauge group; Pati and Salam (1974) proposed another scheme in which hadrons and leptons are placed in a common multiplet.

A: I see. But why do you advocate the four-quark scheme? After all, aren't there other schemes which dispense with the fourth quark?

B: Yes, for example, the Berkeley $\mathrm{M}$-meson model $^{5}$ postulates a large number of scalar mesons which carry both hadronic and leptonic

characteristics. To me, the M-meson dynamics needed to accomplish suppression of the strangeness changing neutral current appears extremely arbitrary and unaesthetic. Besides you recall Weinberg's ${ }^{6}$ remark that the suppression of parity- and strangeness-violations to order $\alpha$ is "unnatural" in theories such as this. Furthermore, models of this type do not seem to lead naturally to an eventual unification of leptons and hadrons. Other models can be constructed, but something is always artificial in them...

A: Well, we seem to be talking about aesthetics, rather than physics substance. By the way, can't you make the fourth quark -- charm, as you call it - - very massive, so that its existence doesn't matter at energies we are, and are likely to be, accustomed to?

B: No, I am afraid not. Clues on the mass of charmed quark come from the study of strangeness changing second-order weak processes, such as $\mathrm{K}_{\mathrm{L}} \rightarrow \mu \bar{\mu}, \mathrm{K}^{+} \rightarrow \pi^{+} \nu \bar{\nu}$ and the $\mathrm{K}_{\mathrm{L}} \mathrm{K}_{\mathrm{S}}$ mass difference. As you know, in a gauge theory of weak and electromagnetic interactions, the 
magnitude of a second order weak amplitude is in general $\mathrm{G}_{\mathrm{F}}{ }^{\alpha},{ }^{1}$ so in order to explain, for example, the observed magnitude of the $\mathrm{K}_{L} \rightarrow \mu \dot{\bar{\mu}}$ amplitude which is of order $G_{F} \alpha^{2}$, we need a suppression mechanism. It is gratifying that in the Weinberg-Salam model the charmed quark, which was invented to remove first order strangeness changing neutral current effects, suppresses higher order, effects as well.

A: Does this mean then that if the charmed quark were degenerate in mass with the usual quarks, there would be no strangeness-changing neutral current effects in any order?

B: Precisely. In any case, in the four-quark version of the WeinbergSalam model the magnitudes of the processes mentioned earlier are all of order $G_{F} \alpha\left(m_{c}^{2}-m_{u}^{2}\right) / m_{W}^{2} \simeq G_{F} \alpha m_{c}^{2} / m_{W}^{2}$ assuming $m_{W}^{2} \gg m_{c}^{2} \gg m_{u}^{2}$, where $m_{c}$ and $m_{u}$ are the masses of the $c-$ and $u$-quarks.

A: So you should be able to make an estimate of $m_{c}$ from the known rates of the aforementioned processes?

B: Yes. The known $K_{L} K_{S}$. mass difference of $0.54 \times 10^{10} \mathrm{nsec}^{-1}$ implies $m_{c}$ of about a few GeV, except that ... .

A: Except what? Pray go on.

B: Well, in the case of $\mathrm{K}_{L} \rightarrow \mu \bar{\mu}$, something extraordinary happens. There are two mechanisms for this decay in second order. One is through $\mathrm{K}_{\mathrm{L}} \rightarrow \mathrm{W}^{+} \mathrm{W}^{-} \rightarrow \mu \bar{\mu}$, the other $\mathrm{K}_{\mathrm{L}} \rightarrow \mathrm{Z} \rightarrow \mu \bar{\mu}$. It turns out that these two diagrams cancel exactly to order $\mathrm{G}_{\mathrm{F}} \mathrm{m}_{\mathrm{c}} \mathrm{m}_{\mathrm{W}} \mathrm{m}^{2}$, so it seems that the amplitude for $K_{L} \rightarrow \mu \bar{\mu}$ is of order $G_{F} \alpha^{2}$ independently of $m_{c}^{\prime}$. A: Hm: That's very interesting. But isn't the cancellation you. referred to very sensitive to the way you treat strong interactions? B: Perhaps. What I have said is based on the calculations of Gaillard and Lee, ${ }^{7}$ who deduced the operator for $\lambda+\bar{n} \rightarrow \mu+\bar{\mu}$ in a free quark model, and then estimated the matrix element of the operator between the $\mathrm{K}_{\mathrm{L}}$ and vacuum states using PCAC. Recently Joglekar(1974) constructed a renormalizable phenomenological model of SU(4) pseudoscalar mesons coupled to Weinberg-Salam gauge bosons. He computed the $\mathrm{K}_{\mathrm{L}} \rightarrow \mu \bar{\mu}$ decay in this model, and found again that this amplitude vanishes to" order $\mathrm{G}_{F} \alpha \mathrm{m}_{\mathrm{c}}^{2} / \mathrm{m}_{\mathrm{W}^{2}}^{2}$, Here $\mathrm{m}_{\mathrm{c}}$ is the mean mass of the charmed pseudo-scalar mesons.

A: That's very intriguing. If I may backtrack, it seems to me an estimate for $\mathrm{K}_{\mathrm{O}} \leftrightarrow \overline{\mathrm{K}}_{\mathrm{O}}$ based on a free quark model is less reliable than that for $\mathrm{K}_{\mathrm{L}} \rightarrow \mu \bar{\mu}$. The point is that for $\mathrm{K}_{\mathrm{O}} \rightarrow \overline{\mathrm{K}}_{\mathrm{O}}$, one should also worry about $\left(\mathrm{W}^{+} \mathrm{W}^{-}+\right.$hadrons $)$as well as the $\mathrm{W}^{+} \mathrm{W}^{-}$intermediate states. So if you discard the quark model calculation for the $K_{L} K_{S}$ mass difference as being unreliable, then there seems to be no need for. a small charmed quark mass. Isn't that right?

B: In a way, yes. But it is hard to imaigine that the quark model calculation of the $K_{L} \dot{K}_{S}$ mass difference is misleading even as to the order of magnitude. Secondly, in the absence of a symmetry argument, the cancsllation of the $K_{L} \rightarrow \mu \bar{\mu}$ amplitude appears purely fortuitous, so 


\section{-8- FERIIIAA B-Pub-74/86-THY}

when strong interactions are taken into account, some suppression of this amplitude may in any case be necessary.

A: By the way, how about $\mathrm{K}^{+} \rightarrow \pi^{+} \nu \bar{\nu}$ ?

B: A good question. For this process, the $\mathrm{W}^{+} \mathrm{W}^{-}$and $\mathrm{Z}$ exchange diagrams do not cancel, and the order of magnitude of the amplitude is

$$
\approx \frac{1}{\pi} G_{F} \times\left(\frac{m_{c}}{38 G_{e V}}\right)^{2} \ln \left(\frac{m_{w}^{2}}{m_{c}^{2}}\right) \sin ^{2} \theta_{c}
$$

in Joglekar's calculation. Here $m_{c}$ is the average mass of the charmed pseudo scalar mesons. Unfortunately the present upper bound on this rate, $\Gamma\left(\mathrm{K}^{+} \rightarrow \pi^{+} \nu \bar{\nu}\right) / \Gamma\left(\mathrm{K}^{+} \rightarrow\right.$ all $)<0.6 \times 10^{-6}:($ Cable, et al. , 1974), implies only a suppression of order $\alpha^{2}$ with respect to the allowed three-body decay:

$$
\Gamma\left(K^{+} \rightarrow \pi^{+\nu}\right) / \Gamma\left(K^{+} \rightarrow \pi^{\circ} e j\right) \leqslant 10^{-5} .
$$

In other words, $\left(m_{c} / 38 \mathrm{GeV}\right)^{2}<1$, which is not too useful.

A: I see. In that case, an improvement of the bound by an order of magnitude or two, short of setting a rate for this process, seems highly desirable.

B: I agree with you completely there.

A: I would think it worthwhile to study the spectroscopy, decay modes and production mechanisms of the charmed particles, assuming their masses are within reach at Fermilab, Super CERN and ISR, or at the next generation of accelerators like PEP, etc., even though I personally am not convinced of their existence.

\section{-9-. FERMILAB-Pub-74/86-TḦ}

B: Thanks, that's precisely what I am working on now.

In the following, we shall interpret "charmed particles" in the narrow sense -- these are particles associated with the fourth quark introduced by Bjorken, Glashow, Iliopoulos and Maiani, and incorporated into the Weinberg-Salam model to banish strangeness-changing neutral currents. In Secs. 2 and 3, we discuss energy levels of low-lying mesonic and baryonic charmed states based on SU(4) - considerations. Sections 4 and 5 deal with; respectively, decay modes and production mechanisms of these particles. Section 6 deals with the possible detection of low-mass charmed particles via their tracks, particularly in emulsions. Section 7 contains a summary and conclusions. 


\section{SPECTROSCOPY}

We shall extend notions of the color triplet quark model of hadrons to the case of four quarks; $u, d, s$ and the charmed c. (Fig. 1a). According to this model, the ground state baryons $\left(\mathrm{J}^{P}=1 / 2,{ }^{+}\right)$are bound states of three quarks, completely antisymmetric in their color indices, and symmetric under the simultaneous interchange of spin and quark labels of any pair. Those states containing only uncharmed quarks $u, d$ and $s$ form the familiar octet of $(1 / 2)^{+}$baryons. Baryon states containing only one charmed quark $c$ may be either symmetric or antisymmetric in the remaining two ordinary quarks. There are altogether six and three such states, respectively. These states carry the charm quantum númber $\mathrm{C}=+1$.

Baryon states with two charmed quarks contain one of the ordinary quarks. That is, there is a triplet of $1 / 2^{+}$baryons with $\mathrm{C}=+2$. These states are listed in Table I.

There are altogether 20 states of $1 / 2^{+}$baryons. They form an irreducible representation $\underset{\sim}{2}$ of $\mathrm{SU}(4) .^{9}$. They form a truncated tetrahadron in the three dimensional plot of $I_{3}, Y$ and $C$ (weight diagram) [ see Fig. 1b]. The truncated tetrahedron has four hexagonal faces, each representing an octet of baryons which transforms irreducibly under an SU(3) subgroup of SU(4), acting on a set of three (out of four ) quarks. Thus, the ordinary baryons, $p, n, \Lambda, \Sigma^{ \pm, 0}, \varrho,-$ form an octet under the sU(3) acting on $u, d$ and $s$; the baryons, $p, n, C_{1}^{++}, C_{1,0}^{+}, C_{1}^{0}, x_{u}^{++}$, $x_{d}^{+}$, for example, form an octet of SU(3) acting on $u, d$ and $c$. This observation turns out to be useful in deducing the $\left(G_{A} / G_{V}\right)$ ratios for weak semileptonic transition from an ordinary nucleon to a charmed baryon (see Sec. 5.2 and Fig. 4).

An inequivalent $20^{\circ}$ of $\mathrm{SU}(4)$ may be found by symmetrizing the three-quark system in SU(4) indices. The weight diagram of this representation (to which one may expect the $3 / 2^{+}$baryons to belong) is a tetrahedron. A three-quark system can also belong to a $\underset{\sim}{\bar{\Psi}}$ of SU(4) (whose weight diagram is an inverted tetrahedron), but this multiplet is not expected to occur in the ground state baryons and will not be ' discussed further.

In this picture mesonic states are formed as bound states of a quark and an antiquark, and we are led to consider 15-plets and singlets of mesons of SU(4). A 15-plet of mesons consists of the usual octet and singlet of $S U(3) \cdot$ with $C^{d}=0, \quad \frac{3}{2}$ and $\overline{3}$ whiç carry $C=+1$ and -1 , respectively. 15-plet singlet [of $S U(4)]$ mixing, as well as octetsinglet mixing of SU(3), depend on the nature of SU(4) breaking. This matter will be discussed at some length in the next section under a set of well-defined dynamical assumptions and what we know about spectroscopy of ordinary $0^{-}$and $1^{-}$mesons. We list these mesons. in Tables II and III; the quark content assignments to neutral mesons, $n, \eta^{\prime}, \eta^{\prime \prime} c^{\prime}$ $\omega, \phi, \phi_{c}$ are approximate, and motivated in Sec. 3. Figure 1c shows the weight diagram of the singlet $+15-$ plet of $S U(4)$, containing the pseudoscalar mesons. 
-12- FERMILAB-Pub-74/ 86-THY

TABLE I

Charmed $1 / 2^{+}$Baryon States

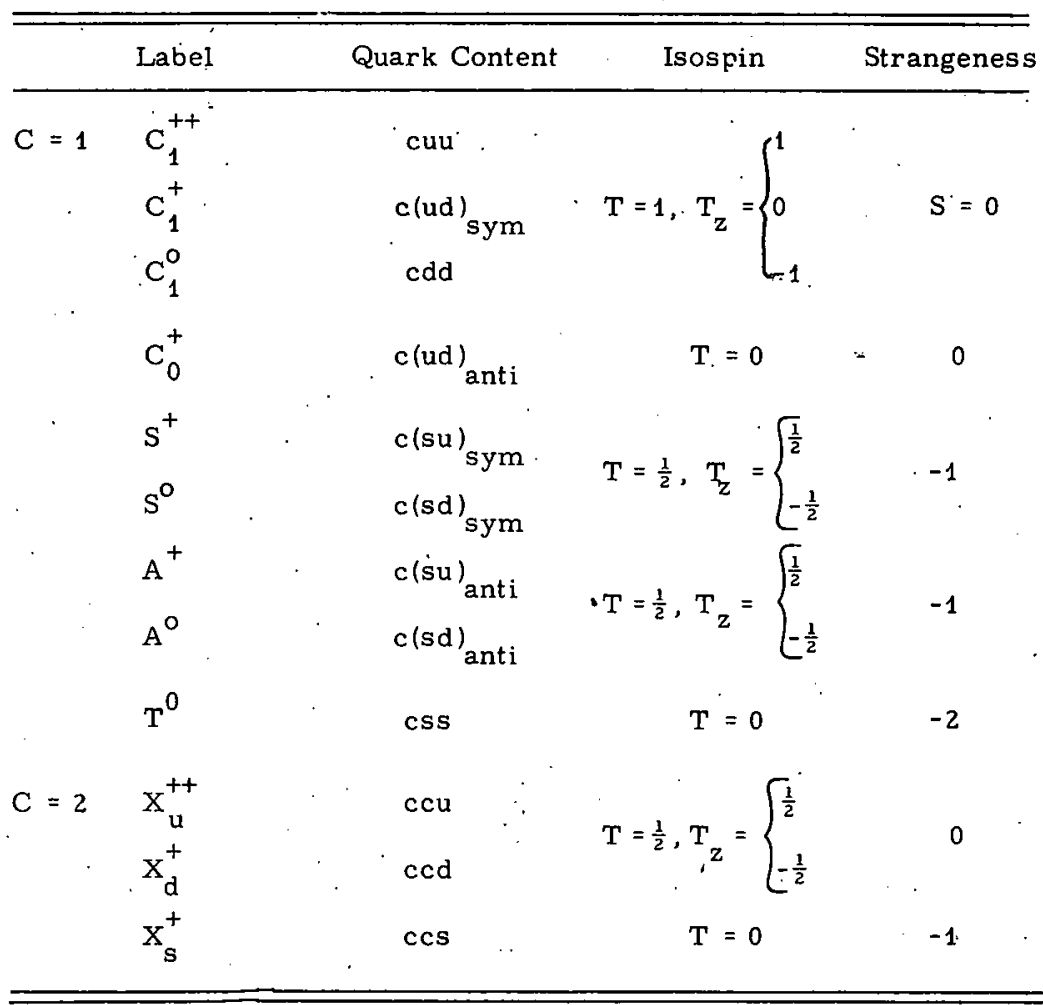

-13- FERMILAB-Pub-74/86-THY

\section{TABLE II}

Charmed $0^{-}$Mesons

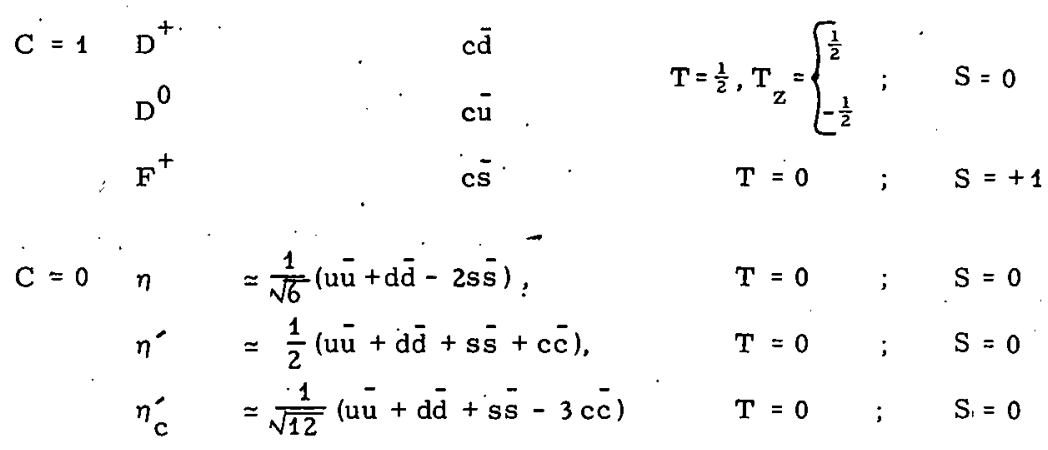

$\begin{aligned} C=-1 & \bar{D}^{0} \\ & D^{-} \\ & F^{-}\end{aligned}$ 
$-14$

TABLE III

Charmed $1^{-}$Mesons

$C=1$

$\omega \simeq \frac{1}{\sqrt{2}}(u \bar{u}-d \bar{d})$

$\phi \simeq \mathrm{s} \overline{\mathrm{s}}$

$\phi_{\mathrm{c}} \simeq \mathrm{c} \overline{\mathrm{c}}$

$C=-1$
$\mathrm{D}^{*+}$

$\mathrm{D}^{* 0}$

$\mathrm{F}^{*+}$

$\bar{D}^{* 0}$

$D^{*-}$

$\mathrm{F}^{*-}$

\section{MASSES OF CHARMED HADRONS}

\subsection{The Nature of SU(4) Symmetry Breaking}

In the spontaneously broken gauge theory.considered here, strong interactions must be invariant under the group $U(4) \otimes U(4)$, except for quark mass terms:

$$
\mathscr{H}_{\text {mass }}=\sum_{i}^{\prime} m_{i} \bar{q}_{i} q_{i} \equiv m_{0} u_{0}+m_{8} u_{8}+u_{1,5} u_{15}
$$

where $m_{0,8,15}$ are linear combinations of the $m_{i}$, and

$$
u_{\alpha} \equiv \bar{q} \lambda_{\alpha} q
$$

The $\lambda_{\alpha}$ are $4 \times 4$ generalizations of the familiar SU(3) matrices; in

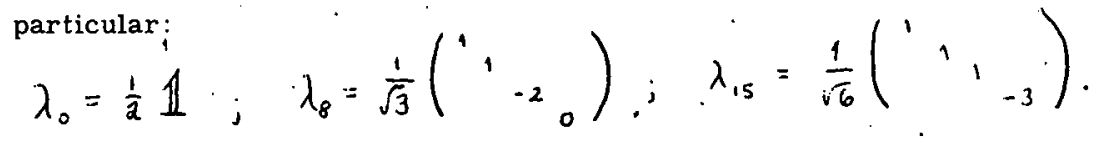

The form of $\mathrm{SU}(4)$ breaking is thus severely restricted, and we may exploit this property in order to gain some insight into the expected mass spectrum of charmed states. We wish to emphasize however that:

a) mass relations derived to lowest order in SU(4) breaking are expected to be much more badly violated than those of SU(3). Nevertheless, they may serve as a useful guide in guessing, for example, whether or not a particular state will decay strongly into a lower mass charméd state ;

b) our predictions are semi-empirical in that we base our 
intuition on the successes of SU(3). An example is the assumption of quadratic mass relations for mesons and linear relations for baryons.

The Hamiltonian density (3.1) may be treated perturbatively on three different levels.

1) SU(4) symmetry breaking. Simple group theoretical arguments allow us to relate the mass of any charmed hadron to the masses of uncharmed states in the same SU(4) multiplet in terms of a single unknown parameter: $\left(\dot{m}_{15} / \mathrm{m}_{8}\right)$ or, equivalently,

$$
R=\left(m_{c}-m_{u}\right) /\left(m_{s}-m_{u}\right)
$$

2) $\mathrm{SU}(4) \otimes \mathrm{SU}(4)$ breaking. To lowest order in chiral $\mathrm{SU}(4)$ breaking, the matrix elements of $u_{0}(S U(4)$ singlet) are related to the matrix elements of $u_{8}$ and $u_{15}$ (15-plet). In general, there is an additional contribution to hadron masses arising from the chiral invariant part of the Hamiltonian; for this reason no additional constraints are obtained except for the pseudoscalar mesons. Pseudoscalar masses are assumed to arise only from the mass term (3.1), and their smallness presumably reflects the smallness of quark masses on a hadronic scale.

This picture is supported by the success of soft pion theorems; low quark masses are also suggested by an analysis of the decay $K_{L} \rightarrow \gamma \gamma{ }^{7}$

3) U(4) $\bigcirc(4)$ breaking. One may also attempt to treat the full symmetry breaking perturbatively. The mass and mixing angle of the SU(4) singlet pseudoscalar are then related to the masses of the 15-plet pseudoscalars. The solution of the relevant equations leads to the prediction of an $\mathrm{I}=\mathrm{Y}=0$ pseudoscalar whose mass satisfies the inequality (Weinberg, 1974b):

$$
m \leqslant \sqrt{3} m_{\pi}
$$

This prediction; which is in flat contradiction with experiment, is independent of the existence of charm. The failure of a perturbative treatment in this case is an outstanding problem of the theory. ${ }^{10}$ Here we simply regard it as an empirical result that the preaking of chiral $\mathrm{U}(4)$ cannot be treated perturbatively.

To lowest order in chiral SU(4) breaking, pseu doscalar meson masses satisfy the relations (Gell-Mann, et al. , 1968, Glashow and Weinberg, 1.968): $\frac{m_{n}^{2}}{m_{l}+m_{u}}=\frac{m_{k}^{2}}{m_{s}+m_{u}}=\frac{m_{D}^{2}}{m_{c}+m_{u}}=\frac{m_{F}^{2}}{m_{c}+m_{s}}$

which directly relate the scale of charmed hadron masșes to quark

masses. In particular, if $m_{u}=m_{d}$, we must have

$$
m_{k}=m_{d} \simeq m_{s} / 2.5 \ll m_{c} \text {. }
$$

The estimate ${ }^{?} m_{c} \lesssim 1.5 \mathrm{GeV}$, together with a lower limit on the $u$ quark mass would provide an upper bound on the charmed particle mass scale. For example, if we assume only that the $u$ and d quarks are heavier than the electron we obtain [ see Eq. (3.2)].

$$
\begin{aligned}
\mathrm{R} & \lesssim 120 \\
\mathrm{~m}_{\mathrm{D}} & \lesssim 5.5 \mathrm{GeV} .
\end{aligned}
$$


One appealing possibility is that since quark and lepton masses arise from the same mechanism -- coupling to Higgs scalars -- their masses may be related (Dittner, et al. , 1973; Eliezer, 1974). If

$$
\mathrm{m}_{\mathrm{d}} / \mathrm{m}_{\mathrm{c}} \simeq \mathrm{m}_{\mathrm{e}} / \mathrm{m}_{\mu}=1 / 200
$$

we obtain:

$$
R \simeq 8, \quad m_{D} \simeq 1.4 \mathrm{GeV}
$$

These values are, of course, purely speculative since even if the chiral SU(4) relation, Eq. (3.3) is approximately valid, we have no real information on quark masses. However, a lower bound of about 1.5 $\mathrm{GeV}$ for charmed hadrons can probably be inferred from the fact that their tracks have not been observed (see Sec. 6). On the other hand, if the mass scale of charmed hadrons is greater than, say, $10 \mathrm{GeV}$, it becomes difficult to understand the very strong suppression of induced strangeness changing neutral currents and of $|\Delta S|=2$ transitions. We shall take as a reasonable range:

$$
8 \leq R \leq 100
$$

i

which corresponds to (approximately!)

$$
1.4 \mathrm{GeV} \lesssim \mathrm{m}_{\mathrm{D}} \lesssim 5 \mathrm{GeV}
$$

for the lowest pseudoscalar state and (see below)

$$
2.4 \mathrm{GeV} \lesssim \mathrm{m}_{\mathrm{C}_{0}} \lesssim 19 \mathrm{GeV}
$$

for the lowest baryon state. In the remainder of this section we shall display SU(4) mass formulae for baryons and mesons às a function of the scale parameter $R$. There is also the possibility, of course, that the calculation of the charmed quark mass ${ }^{7}$ does not constrain charmed particle masses. Nonetheless, we would regard the absence of any charmed particle below $10 \mathrm{GeV}$ as a serious argument against such a scheme.

\subsection{Pseudoscalar Mesons}

To lowest order in the SU(4) symmetry breaking term (3.1), the meson masses may be described by an effective Lagrangian of the form:

$$
\begin{aligned}
\mathscr{L}_{\text {mass }}= & \mu_{0}(\operatorname{Tr} \pi)^{2}+\mu_{1} \cdot \operatorname{Tr}\left(\pi^{2}\right)+ \\
& \alpha \operatorname{Tr}(\pi \Delta \pi)+\beta(\operatorname{Tr} \pi)(\operatorname{Tr} \Delta \pi),
\end{aligned}
$$

where $\pi$ is the $4 \times 4$ matrix representation of the pseudoscalar states (15-plet plus singlet) and $\Delta$ is a traceless diagonal matrix with two independent elements : $m_{s}-m_{u}, m_{c}-m_{u}$. There are five independent parameters in expression (3.4): $\mu_{1}$, the "mean" meson mass; $\mu_{0}$, which separates the SU(4) singlet from the 15-plet; $\alpha\left(m_{s}-m_{u}\right)$ and $\alpha\left(m_{c}-m_{u}\right)$ which determine the mass shifts of strange and charmed states,

respectively; and

$$
\gamma \equiv \dot{\beta} / \alpha
$$

which determi:1es the singlet-15-plet mixing. In group theoretical 
language, these five,parameters correspond to four independent reduced matrix elements:

$$
\langle 1|| 1|| 1\rangle,\langle 15 \| 1|| 15\rangle,\langle 15|| 15|| 15\rangle,\langle 1|| 15|| 15\rangle
$$

and the mass difference ratio $\mathrm{R}[\mathrm{Eq} .(3, .2)]$.

Since four independent masses $\left(\pi, \eta, K, x^{0}\right)$ are known from experiment, we may eliminate four parameters and express the remaining masses in terms of the scale parameter $R$.

For states which do not mix with the SU(4) singlet we obtain:

$$
\mathrm{m}_{\mathrm{D}}^{2}-\mathrm{m}_{\pi}^{2}=\mathrm{m}_{\mathrm{F}}^{2}-\mathrm{m}_{\mathrm{K}}^{2}=\mathrm{R}\left(\mathrm{m}_{\mathrm{K}}^{2}-\mathrm{m}_{\pi}^{2}\right)
$$

Now consider the mixing of $\mathrm{I}=\mathrm{Y}=0$ states $\left(\eta, \eta^{\prime}\right.$ and $\eta_{\mathrm{c}}^{\prime}$ in Table II).

There are two values of the mixing parameter $\gamma$ which are of particular " physical interest.

a) The value $\gamma=0$ separates states according to the masses of their constituents in the limit $\mu_{0} \rightarrow 0$. For finite $\mu_{0}$ but $R \gg 1$, this choice effectively separates out the $(\vec{c})$ state which becomes much heavier than the others. However, fixing $\gamma$ also fixes the SU(3) octet-singlet mixing as a function of pseudoscalar meson masses, and the choice $y \simeq 0$ does not allow a fit to the observed masses $\left[\eta(549)\right.$ and $\left.\eta^{-}(958)\right]$.

b) The value $y_{1}=-\frac{1}{2}$ separates the $S U(4)$ singlet $\left(\eta^{\prime} \equiv \operatorname{Tr} \pi\right.$ ) from the 15-plet. The $\eta, \eta_{c}^{\prime}$ mixing is then determined to be very small:

$$
\begin{aligned}
& \eta^{\prime} \eta^{\prime} \varepsilon \eta_{c}^{\prime} \\
& \eta_{c}^{\prime} \rightarrow \eta_{c}^{\prime}-\varepsilon \eta \\
& \varepsilon \simeq 2 \sqrt{2} / 9 R \ll 1
\end{aligned}
$$

for $R \geq 8$. The $\eta_{\mathrm{c}}^{\prime}$ mass is given (to order $\epsilon^{2}$ by):

$$
m_{n_{c}^{\prime}}^{2}=m_{\pi}^{2}+\frac{3}{2}\left(R+\frac{1}{9}\right)\left(m_{K}^{2}-m_{\pi}^{2}\right) \simeq \frac{3}{2} m_{D}^{2} .
$$

If we now wish to account for the deviation of the $\eta$ mass from the GellMann-Okubo relation

$$
m_{n}^{2}=\left(4 m_{K}^{2}-m_{\pi}^{2}\right) / 3
$$

by a small $\eta, \eta^{\prime}$ and/or $\eta, \eta_{c}^{\prime}$ mixing, we may allow

$$
Y+\frac{1}{2} \equiv 2 \delta \neq 0 \text {. }
$$

Then we obtain to lowest order in the mixing:

$$
\eta_{i} \rightarrow \eta_{i}+\mathcal{E}_{i j} \eta_{j}\left(\frac{m_{k}^{2}-m_{\pi}^{2}}{m_{j}^{2}-m_{i}^{2}}\right)
$$

with

$$
\begin{aligned}
& \varepsilon_{22^{\prime}}=\sqrt{213} \delta, \quad \varepsilon_{2 \eta_{c}^{\prime}}=\sqrt{2} / 3 . \\
& \varepsilon_{\eta^{\prime} \eta_{c}^{\prime}}=-\sqrt{3} \delta(R-1 / 3) / 2 .
\end{aligned}
$$

The mixing parameter $\delta$ is determined as a function of the physical masses by:

$$
\delta^{2}=\frac{3}{2} \frac{\left(m_{l}^{2}-m_{l}^{2}\right)}{\left(m_{k}^{2}-m_{n}^{2}\right)^{2}}\left\{\frac{4 m_{r}^{2}-m_{n}^{2}}{3}-m_{l}^{2}-\frac{2}{9} \frac{\left(m_{k}^{2}-m_{\pi}^{2}\right)^{2}}{\left(m_{l_{l}^{2}}^{2}-m_{l}^{2}\right)}\right\} .
$$


Positivity of $\delta^{2}$ requires:

$$
m_{\eta_{c}^{\prime}}^{2} \gtrsim 930 \mathrm{MeV} .
$$

A priori one could identify $\eta_{\mathrm{c}}^{\prime}$ with the observed state at $958 \mathrm{MeV}$, in which case the $\mathrm{SU}(4)$ singlet is unmixed $1 \delta \approx 0$ ) and need not even exist. This is the solution originally discussed by Bjorken and Glashow(1964); however, the masses in Eq. (3.7) are then determined to be unacceptably low. For $R \geq 8$, the $\eta, \eta_{c}^{\prime}$ mixing becomes negligible, and the $\eta, \eta^{\prime}$ mixing reduces to the usual treatment.

\section{3 Vector Mesons}

The treatment for vector mesons is similar to that for pseudoscalars, with the masses again described by a phenomonological Lagrangian of the form (3.4). However, in this case, the solution chosen by nature is

$$
\mathrm{v}=\mu_{0}=0
$$

so that states separate according to their heavy quark content as indicated in Table III. The SU(4) mass relations are:

$$
m_{D^{\star}}^{2}-m_{p}^{2}=m_{F^{*}}^{2}-m_{K^{*}}^{\ddot{2}}=\frac{1}{2}\left(\dot{m}_{\varphi_{e}}^{2}-m_{\rho}^{2}\right)=R\left(m_{k^{*}}^{2}-m_{p}^{2}\right) \text {. }
$$

\subsection{Spin 1/2 Baryons}

The masses of the ground state baryon multiplet (Table I), depend on three reduced matrix elements: One for the $\mathrm{SU}(4)$ singlet operator and two for the 15 operator, corresponding to symmetric (d) and antisymmetric (f) couplings. The values of these parameters can be

inferred from the known baryon masses, and the following relations are obtained.

$$
\begin{aligned}
& m_{c_{1}}-m_{p}=m_{T}-m_{\equiv}=m_{S}-\left(\frac{3 m_{1}+m_{\Sigma}}{4}\right)=R\left(m_{\Sigma}-m_{p}\right), \\
& m_{c_{0}}-m_{P}=m_{A}-\left(\frac{3 m_{\Sigma}+m_{i}}{4}\right)=R\left(m_{\Lambda}-m_{p}\right), \\
& m_{x_{u, d}}-m_{p}=m_{x_{s}}-m_{\Sigma}=R\left(m_{\equiv}-m_{p}\right) .
\end{aligned}
$$

In the above relations we have neglected the mixing of $S$ and $A$ which arises from SU(3) breaking. This effect is in fact negligible since $\mathrm{SU}(3)$ breaking is very small on the charm mass scale. If $\mathrm{B}_{3}$ and $\mathrm{B}_{6}$ denote states which transform according to irreducible representations of SU( 3$)$, the physical states are:

$$
\begin{gathered}
A \simeq B_{\overline{3}}+\mathcal{E} B_{6} \\
S \simeq B_{6}-\varepsilon B_{\overline{3}} \\
\varepsilon \simeq(4 R-2)^{-1} \lesssim 0.03
\end{gathered}
$$

for $R \geq 8$. The effect of the mixing on the masses is second order in $\epsilon$ 


\section{DECAYS OF CHARMED PARTICLES}

\subsection{Mesons :- Leptonic Decays}

Leptonic decays of charmed mesons can be estimated with some confidence since the structure of the hadronic charged current is given by the Weinberg-Salam theory:

$$
\begin{aligned}
J_{\mu}^{c} & =\pi f_{\mu}\left(1-y_{s}\right)\left(d \cos \theta_{c}+s \sin \theta_{c}\right) \\
& +\bar{c} y_{\mu}\left(1-f_{s}\right)\left(-d \sin \theta_{c}+s \cos \theta_{c}\right)
\end{aligned}
$$

In the following discussion we shall neglect form factors, although they may have more rapid variation than in the case of $K$ decay. This is due to the fact that the vector-pseudoscalar mass splitting is expected to be smaller for charmed particles. Then, for example in the decay $\mathrm{D} \rightarrow \mathrm{K} \ell v$ a form factor $f\left(q^{2}\right)=m_{D}^{2} *\left(m_{D}^{2}-q^{2}\right)$ with $0 \leqslant q_{t}^{2} \leqslant\left(m_{D}-m_{K}\right)^{2}$ could lead to an appreciable enhancement with respect to the estimate given below.

An important selection rule emerges from the structure of the charm-changing current in (4.1);

$$
\Delta Q=\Delta C=\Delta S, \Delta I=0 \text {, with } \cos \theta_{c} \text { in amplitude }
$$

or

$$
\Delta Q=\Delta i, \Delta S=0 ; \Delta I=1 / 2 \text { with } \sin \theta \text { in amplitude }
$$

The dominant decay modes $\left(\sim \cos \theta_{c}\right)$ are represented schematically in Fig. $2 a$.
4.1.1 Two-body decays. These are analogous to the $K_{\ell 2}$ decays. We define $f_{D}$ and $f_{F}$ by

$$
\begin{aligned}
& \left\langle 0\left|J_{\mu}^{c}\right| D^{+}(q)\right\rangle=i f_{D} q_{\mu} \sin \theta_{c} \\
& \left\langle 0\left|J_{\mu}^{c}\right| F^{+}(q)\right\rangle=-i f_{F} q_{\mu} \cos \theta_{c}
\end{aligned}
$$

If the $S U(4)$ symmetry is not spontaneously broken, we expect that

$$
f_{D} \simeq f_{F} \simeq f_{K} \simeq f_{\pi}
$$

Thus we obtain

$$
\frac{\Gamma\left(D^{+} \rightarrow \mu^{+\nu}\right)}{\Gamma\left(K^{+} \rightarrow \mu^{+} \nu\right)} \simeq\left(\frac{m_{0}}{m_{k}}\right)
$$

and

$$
\frac{\Gamma\left(F^{+} \rightarrow \mu^{+\nu}\right)}{\Gamma\left(K^{+} \rightarrow \mu^{+\nu}\right)} \simeq\left(\frac{m_{F}}{m_{k}}\right) \cot ^{2} \theta_{c}
$$

With $\Gamma\left(\mathrm{K}^{+} \rightarrow \mu^{+} \nu\right) \simeq 0.5 \times 10^{8} \mathrm{sec}^{-1}$, we predict

$$
\begin{aligned}
& \Gamma\left(D^{+} \rightarrow \mu^{+} \nu\right) \simeq \frac{1}{2}\left(\frac{m_{D}}{m_{k}}\right) \times 10^{8} \mathrm{sec}^{-1} \\
& \Gamma\left(F^{+} \rightarrow \mu^{+} \nu\right) \simeq 0.9\left(\frac{m_{F}}{m_{k}}\right) \times 10^{9} \mathrm{sec}^{-1}
\end{aligned}
$$

4.1.2. Three-body decays. An example is $\mathrm{D}^{+} \rightarrow \mathrm{K}^{0} \ell^{+} v$, which is analogous to $\mathrm{K}^{0} \rightarrow \pi^{-} \mathrm{e}^{+} \nu$. To the extent that the lepton mass is negligible in comparison to the $Q$-value, we may neglect the second form factor $f_{-}$. Assuming the $f_{+}$form factors are approximately constant and are equal for the $\mathrm{D}_{\ell 3}$ and $\mathrm{K}_{\ell 3}$ decays, we have 


$$
\begin{aligned}
& \frac{\Gamma\left(D^{+} \rightarrow \bar{K}^{\circ} l^{ \pm}-\nu\right)}{\Gamma\left(K^{0} \rightarrow \pi^{-} e^{+\nu}\right)} \simeq\left(\frac{m_{C}}{m_{k}}\right)^{6} \frac{f\left(x_{D}\right)}{f\left(x_{k}\right)} \cot ^{2} \theta_{c}
\end{aligned}
$$

where

$$
\begin{aligned}
& f(x)=1-8 x^{2}+8 x^{6}-x^{8}-24 x^{4} \ln x \\
& x_{D}=\left(m_{k} / m_{D}\right) \text { and } x_{k}=\left(m_{\pi} / m_{k_{1}}\right)
\end{aligned}
$$

For $m_{D} \simeq 1.5 \mathrm{GeV}$, we have $x_{D} \simeq x_{K}$ for $m_{D} \rightarrow \infty$, the ratio $f\left(x_{D}\right) / f\left(x_{K}\right)$ is about 2 .

$$
\begin{aligned}
& \text { Since } \Gamma\left(K^{0} \rightarrow \pi^{-} e^{+} \nu\right)=\Gamma\left(K_{L} \rightarrow \pi e v\right) \simeq 10^{7} \mathrm{sec}^{-2}, \text { we expect } \\
& -\Gamma\left(D^{+} \rightarrow \bar{K}^{\circ} l^{+} \nu\right)=\Gamma\left(D^{\circ} \rightarrow K^{-} l^{+} \nu\right) \\
& \simeq 0.5\left(\mathrm{~m}_{D} / \mathrm{GeV}\right)^{6} \times 10^{10} \mathrm{sec}^{-1}
\end{aligned}
$$

Thus for $m_{D}=2 \mathrm{GeV}$ and for two kinds of leptons $\mu, e$, we, expect

$$
P\left(D^{+} \rightarrow \overline{K^{0}} \mu^{+\nu} \text { and } \overline{K^{0}} e^{+\nu}\right) \simeq 3 \times 10^{11} \mathrm{sec}^{-1}
$$

In addition, there are decays into nonstrange final states, such as $\mathrm{D}^{+}=\pi^{0} \mu^{+} \nu$. These decays, however, are suppressed by a factor $\tan ^{2} \theta_{c} \simeq 0.05$ compared to decays into $K$ mesons.

Three-body semileptonic decays of the $\mathrm{F}^{+}$mesons are more complex. The main decay modes are

$$
\begin{aligned}
F^{+} & \rightarrow \eta+l^{+}+\nu \\
& \rightarrow x^{0}+l^{+}+\nu \\
& \rightarrow K^{0}+l^{+}+\nu
\end{aligned}
$$

Presumably, the first decay mode is the most important one, and we

estimate

$$
\frac{\Gamma\left(F^{+} \rightarrow \eta l^{+} \nu\right)}{\Gamma\left(k_{L} \rightarrow \pi e^{+\nu}\right)} \simeq \frac{2}{3} \cot ^{2} \theta_{c}\left(\frac{m_{F}}{m_{k}}\right)^{5}
$$

$$
\left.\Gamma\left(F r \rightarrow \eta \mu^{+\nu}\right) \text { and } \eta e^{+\nu}\right) \simeq 7 \times\left(m_{F} / \mathrm{GeV}^{5} \times 10^{9} \mathrm{sec}^{-1}\right.
$$

4.1.3. Multipion decays. In addition to the decays discussed above, there will be decays in which hadronic final states contain many pions, such as

$$
D^{0} \rightarrow \bar{k}+n \pi+l^{+}+2 .
$$

If the mass of the charmed meson in question is large enough, one expects the decays into multipion final states to occupy a significant fraction of the total rate. However, the re is a reason to think that perhaps multipion decays are less frequent than one would guess at first. This is that in the soft pion limit of any of the final state pions, the amplitude vanishes:

$$
\begin{aligned}
\lim _{k \rightarrow 0} & \left\langle\bar{k} \cdots \pi^{\alpha}(k)\left|\bar{s} f_{\mu}\left(1-\gamma_{5}\right) e\right| D^{0}\right\rangle \\
& =\frac{1}{i f_{\pi}}\left\langle\bar{k} \cdots\left|\left[\bar{s} \gamma_{\mu}\left(1-\dot{\gamma}_{5}\right) c, Q_{\sigma}^{\alpha}\right]\right| D^{c}\right\rangle \\
& =0
\end{aligned}
$$

4.1.4. Inclusive semi-leptonic estimate. We view semileptonic 
decays of a charmed particle as occuring due to the elementary processes $c \rightarrow s+l^{+}+v, c \rightarrow d+l^{+}+v$, followed by deexcitation of the remnant of the hadronic matter which results from replacing the initial charmed quark by either $s$ or $d$. If we sum over all final states, the rate of semileptonic decays should be given essentially by the elementary process, provided that the mass of the charmed quark is sufficiently large so that the hadronic state which follows lepton-pair emission has a $100 \%$ probability to decay into stable hadrons. If this is true, then the total semileptonic decay rate of a charmed particle is given by the same formula as for $\mu$-decay:

$$
\Gamma_{\text {tutal }}\left(\text { charm } \rightarrow S_{\nu}+\text { hadrons }\right)=\frac{G_{i=}^{2} m_{i}^{5}}{196 \pi^{3}}
$$

where $m_{c}$ is the mass of the c-quark. In.footnote 7 , the value $m_{c} \simeq 1.5 \mathrm{GeV}$ was suggested. This implies (for summing over leptons) $\Gamma_{\text {total }}\left(\right.$ charm $\rightarrow(y+h a d r e n s): 10^{12} \mathrm{sec}^{-1}$

A similar estimate can be made on the total nonleptonic decay rate of a charmed particle (see Sec, 4.2.1., below); we find that in this simple-minded model

$$
\frac{\Gamma_{\text {total }}(\text { charm } \rightarrow(\nu+\text { hadrous })}{\Gamma_{\text {total }} \text { (charm } \rightarrow \text { hadrons) }} \because 2 \tan ^{2} \theta_{\text {c }} \sim 8 \%
$$

\subsection{Mesons - Nonleptonic}

To lowest order nonleptonic decays of charmed particles are induced by a term in the current $\mathbf{x}$ current interaction:

$$
\begin{gathered}
\mathscr{H}_{w}^{\prime \prime}=\frac{G_{i^{x}}}{\sqrt{2}} \cos ^{2} \theta_{c}\left\{\dot{u} \gamma_{\mu}\left(1-\gamma_{s}\right) d \bar{s} \gamma^{\mu}\left(1-\gamma_{\dot{x}}\right) c\right. \text { th.e. } \\
\left.+\theta\left(\tan \theta_{c}\right)\right\}
\end{gathered}
$$

While we do know the form of the interaction responsible for charmed particle decays, we are not in la position to predict their decays reliably, since doing so would entail complete command of hadron dynamics. So the following discussion should only be considered as an educated guess.

We know that some sort of enhancement is necessary to account for the magnitude of nonleptonic decays of ordinary hadrons, and, in particular, the $\Delta \mathrm{I}=\frac{1}{2}$ (or octet) rule. According to results of Gaillard and Lee (1974b) and of Altarelli and Maiani (1974), such an enhancement can arise in color quark models as a renormalization effect due to color glụn exchange. We may extend this argument to the charm decay interaction (4.11) and find that charm decays should also be enhanced, to the same extent as the enhancement of the $\Delta I=\frac{1}{2}$ part of strange particle decays. Thus, we find that the effective operators responsible for charm particle decays are bigger by $\cos \theta_{c}$ than that for strange particle decays. With this in mind, we shall make sevèral guesses.

4.2.1. Quark Model. The total width of a charmed meson must 
be proportional to $\left(A G_{F} \cos ^{2} \theta_{c}\right)^{2}$ where $A$ is the enhancement factor alluded to. From phenomenological analyses of nonleptonic decays of ordinary hadrons, we estimate $A \cos \theta_{C} \sin \theta_{C} \simeq 1$.

Now consider a charmed particle as a collection of quarks confined in a finite space region by any. one of the mechanisms recently proposed." The nonleptonic decay of this state will be triggered by the process $c \rightarrow s+u+d$ followed by breakup of the confinement (bag) into many stable hadrons. If the geometrical size of the bag is sufficiently large compared to the wavelengths of the final state quarks, so that the density of available final states is nearly equal to the case of a "free" charmed quark decay, the total rate of nonleptonic decays. of a charmed particle is given by the rate of the elementary process $c \rightarrow s+u+\bar{d}$. If the mass of $c$ is much bigger than those of the other quarks, the rate for the latter is given by the same formula as for $\mu$-decay:

$$
\begin{aligned}
\left.\Gamma_{\text {total }} \text { (charm } \rightarrow \text { hadrons }\right) & =\frac{1}{196 \pi^{3}}\left(G_{F} A \cos \theta_{c}\right)^{2} m_{c}^{5} \\
& =18\left(\frac{m_{c}}{m_{\mu}}\right)^{5} \Gamma(\mu \rightarrow \mathrm{e} \nu \bar{v}) \\
& \approx\left(\frac{m_{c}}{m_{\mu}}\right)^{5} \times 10^{7} \cdot \mathrm{sec}^{-1}
\end{aligned}
$$

where $m_{c}$ is the mass of the charmed quark. Assuming $m_{c}=1.5 \mathrm{GeV}$ as suggested by Gaillard and Lee (1974a), we. guess that.

$$
\Gamma_{\text {total }}(\text { charm } \rightarrow \text { hadrons })=10^{13} \mathrm{sec}^{-1} \text {. }
$$

Just as $f$-values of $\beta$ transitions of nuclei vary widely the total

decay rates of charmed particles may vary from the above estimate by as much as a few orders of magnitude either way. (The same remark applies to the estimate of the total leptonic rates of Sec. 4.1.4.)

4.2.2. A specific two-body final state Let us consider, as an illustration, the process $\mathrm{D}^{0} \rightarrow \mathrm{K}^{-}+\pi^{+}$. We estimate the amplitude of this decay by the following approximation:

$$
\begin{aligned}
& T\left(D^{0} \rightarrow K^{-} \pi^{+}\right) \simeq \\
& \frac{G_{F}}{\sqrt{2}} \cot \theta_{c}\left\langle\pi+1 \bar{u} f_{\mu}\left(1-\gamma_{5}\right) d \mid 0\right\rangle\left\langle K^{-}\left|\bar{s} f^{\mu}\left(1-\gamma_{s}\right) c\right| D^{\circ}\right\rangle
\end{aligned}
$$$$
\simeq \frac{G_{F}}{\sqrt{2}} \cot \theta_{c} f_{\pi} m_{0}^{2} \text {. }
$$

Thus

$$
\begin{aligned}
\Gamma\left(D^{0} \rightarrow K^{-} \pi^{+}\right) & \simeq \frac{1}{2 m_{D}} \frac{1}{2} G_{F}^{2} \cot ^{2} \theta_{C} \cdot f_{\pi}^{2} m_{D}^{4} \frac{1}{8 \pi} \\
& =G_{F}^{2} f_{\pi}^{2} m_{D}^{3} \frac{1}{32 \pi} \cot ^{2} \theta_{C} \\
& \simeq 6 \times\left(m_{D} / G_{e} v\right)^{3} \times 10^{11} \mathrm{sec}^{-1},
\end{aligned}
$$

so we expect, for $\mathrm{m}_{\mathrm{D}}=2 \mathrm{GeV}$

$$
\Gamma\left(D^{\circ} \rightarrow K^{-} \pi^{r}\right) \simeq 5 \cdot 10^{12} \mathrm{sec}^{-1} .
$$

Note that the above formula scales only as $\mathrm{M}_{\mathrm{D}}{ }^{3}$, in contrast to the semileptonic rate for $\mathrm{D}^{+} \rightarrow \mathrm{K}^{0} \ell^{+}{ }^{+}$, estimated above which scales as $\mathrm{m}_{\mathrm{D}}^{5}$. For very large $M_{D^{\prime}}$ as will be shown below, the multi-pion final states are extremely important in nonleptonic decays, and one thus can expect a comparable $M_{D}^{5}$ scaling for nonleptonic decays as well.

An exception to the general enhancement rule may occur when the nonleptonic decay leads to an exotic final state, as in the case of the $\cos ^{4} \theta_{C}$ decay of the $\mathrm{D}^{+}$:

$$
D^{+} \rightarrow(\bar{K} m i)^{+}
$$


where $m=1,2, \ldots$

We have seen such behavior in the decays $K^{ \pm} \rightarrow \pi^{ \pm} \pi^{0}$, where the $\pi \pi$ system must have $I=2$. Such decays seem to lack the enhancement factor $A$.

Note that $: \Delta C=\Delta S$ nonleptonic decays obey the rule $|\Delta I|=1$. Thus two-body decays of $\mathrm{F}^{+}$consist of $\mathrm{F}^{+} \rightarrow \mathrm{K}^{+} \overline{\mathrm{K}}^{0}$ and $\eta \pi^{+}$. We guess that all two-body nonleptonic decays of charmed $0^{-}$mesons proceed at the rate of approximately $(\mathrm{M} / \mathrm{GeV})^{3} \times 10^{11} \mathrm{sec}^{-1}$.

4.2.3. "Statistical" model. To estimate the total hadronic (i.e., nonleptonic) decay, rate, we shall take the following simple model. We assume that the amplitudes for $\mathrm{D}^{0} \rightarrow \overline{\mathrm{K}}^{+} \mathrm{n} \pi$ are independert of external momenta and adopt the following current-algebra inspired guess:

$\ldots P(D \rightarrow \bar{K}+n \pi)=\frac{c}{f_{\pi}^{2 n}} \int d \operatorname{LPS}\left(m_{D}^{2} ; K, P_{1}, \ldots, P_{n}\right)$.

With the notation

$$
\Gamma(D \rightarrow \bar{K}+n \pi)=\Gamma_{n}
$$

we find that

$$
\frac{\Gamma_{n+1}}{\Gamma_{n}} \simeq\left(\frac{m_{D}^{2}}{4 \pi \cdot f_{\pi}}\right)^{2} \frac{1}{n(n+1)}
$$

In Table IV, we give branching ratios of $\overline{\bar{K}}+n \pi$ as functions of the mass $\mathrm{m}_{\mathrm{D}}$. According to this table, the total nonleptonic decay rate is expected to be

$$
\Gamma_{\text {rotal }}(\text { charm } \rightarrow \text { hadrons }) \simeq \text { a few } \times 10^{12} \mathrm{sec}^{-1}
$$

if the mass of the charmed meson is $\simeq 2 \mathrm{GeV}$. (Note that as $\mathrm{m}_{\mathrm{c}}$ increases, the assumption of a constant matrix element becomes more dubious and is made here only for an order of magnitude estimate: )

$$
\text { 4. 2.3. } \mathrm{D}^{0}-\mathrm{D}^{-0} \text { mixing. Just as } \mathrm{K}^{0} \text { and } \bar{K}^{0} \text { mix to produce } \mathrm{K}_{1}
$$
and $K_{2}$ as two states with definite lifetime, so do $D^{0}$ and $\bar{D}^{\circ}$ mix. This comes about from the circumstance that both $D^{0}$ and $\bar{D}^{0}$ decay into $2 \pi, \dot{3} \pi, \ldots$ states of zero strangeness. However these decay amplitudes are proportional to $\sin \theta_{c}$. Thus, the real and imaginary off-diagonal elements of the $D^{0} \bar{D}^{-0}$ mass matrix are expected to be suppressed by a factor of $\tan ^{2} \theta_{c}$ relative to diagonal ones. While

$$
\begin{aligned}
& D_{1}=\left(D^{0}-\overline{D^{0}} j i \sqrt{2} i\right. \\
& D_{2}=\left(D^{0}+\bar{D}^{0}\right) / \sqrt{2}
\end{aligned}
$$

are eigenstates of the mass matrix, the lifetimes $\Gamma_{1}$ or $\Gamma_{2}$ would be very close to each other, and the mass difference $\Delta \mathrm{m}=\mathrm{m}_{1}-\mathrm{m}_{2}$ would be small compared to $\Gamma_{1}$ or $\Gamma_{2}$. This means that $D^{0}$, when it is produced, would decay mostly into $\Delta S=-1$ channels, before it can turn into $\bar{D}^{0}$. Under these circumstances, then, the effects of $D^{0}-\bar{D}^{0}$ mixing are not very important in decays of these particles.

\subsubsection{Mass spectra and exotic combinations. The nonleptonic} decays arising from $c \rightarrow$ uds (or $c \tilde{u} \rightarrow \bar{d} s, c s \rightarrow u \bar{d}, c d \rightarrow$ us, etc.) proceed with rates proportional to $\cos ^{4} \theta_{c}$. These decays are thus the favored ones. They are illustrated in Fig. $2 b$.

Explicitly, one expects the following final states from nonleptonic $\cos ^{4} \theta_{c}$ decays: 


$$
\begin{aligned}
& D^{0} \rightarrow k^{-} \pi^{+}, \overline{k^{0}} \pi^{-} \pi^{r}, \ldots \\
& F^{+} \rightarrow \pi^{-} \pi^{+} \pi^{+}, k^{-} k^{+} \pi^{+}, \ldots \\
& D^{+} \rightarrow k^{-} \pi^{+} \pi^{+}, \ldots
\end{aligned}
$$

Note that the last state is exotic. (This circumstance, as mentioned earlier, may lead to the relative suppression of the nonleptonic' decays of $\mathrm{D}^{+}$, in analogy with the case of $\mathrm{K}^{+} \rightarrow \pi^{+} \pi^{0}\left(\mathrm{I}_{\pi \pi}=2\right)$ versus $\left.K_{S}^{0} \rightarrow \pi^{+} \pi^{-} \cdot\left(I_{\pi \pi}=0\right)^{12}\right)$. Note also that to order $\cos ^{4} \theta_{C}$ the nonleptonic decays of $q \bar{q}$ charmed mesons do not lead to states with the quantum numbers of $K^{ \pm}$. The presence of a narrow peak in a $(\mathrm{Km} \pi)^{0}$ or $(\overline{\mathrm{K} m} \pi)^{0}$ distribution and its absence in $(\mathrm{Km} \pi)^{+}$or $(\overline{\mathrm{K}} \mathrm{m} \pi)^{-}$distributionș would thus be alstrong indication in favor of charmed particles.

The transitions $c \rightarrow$ udd, $c \rightarrow$ uss, etc:, can occur to order $\cos ^{2} \theta_{c}$ $\sin ^{2} \theta_{c}$. The final states to which they lead are shown in Fig. 2c.

\subsection{Baryon Decays.}

Charmed baryons may decay weakly according to the selection rules discussed above (valid to order $\sin ^{2} \theta_{c}$ ):

$$
\Delta C=\Delta S=\Delta Q,|\Delta I|=O \quad \mid \quad \text { (4.12) }
$$

for leptonic decays, and

$$
\Delta C=\Delta S=-\Delta I_{3},|\Delta I|=1
$$

for non-leptonic decays. In this case they would appear as very sharp resonances in a variety of channels, some owith exotic quantum numbers:

$$
\begin{aligned}
T^{0} \rightarrow \dot{\Omega}^{-} \pi^{+}, & S^{+} \rightarrow \Xi^{0} \pi^{+}, \\
& C_{1}^{++} \rightarrow \Delta^{++} \overrightarrow{K^{0}}, \Sigma^{+} \pi^{+}
\end{aligned}
$$

etc.
However if the mass formulae of Sec. 3 have any relevance, it is more likely that charmed baryons will undergo charm conserving strong decays through cascade procésses as illustrated in Fig. 3. This expectation is, a direct consequence of the assumptions of linear mass formulae for baryons and quadratic formulae for mesons: Charmed baryon masses grow linearly with $R$ and meson masses grow as $R^{\frac{1}{2}}$. Since our intuition here may be wrong (and also because the mass predictions can be badly violated), one should not exclude the possibility that at least some of the charmed baryons may be stable against strong decay.

Nevertheless we are faced with the prospect of a strong and weak decay chain in which charmed particles will appear as broad resonances in multiparticle channels. If masses are relatively low, the dominant decay modes will probably be of the type:

$$
B_{c} \rightarrow B+P_{c}
$$

Transition rates can be estimated using $\mathrm{SU}(4)$ to relate the couplings to the pion nucleon coupling constant. We assume $f / d \simeq 0.6$ as suggested by PCAC and data on semi-leptonic baryon decays. The partial width is then given by:

$$
\Gamma\left(B \rightarrow B^{\prime}+P\right)=\frac{g_{B B^{\prime}}^{2} P}{16 \pi}\left\{\frac{(m-m)^{2}-\mu^{2}}{M^{2}}\right\}^{3 / 2}\left\{(m+m)^{2}-\mu^{2}\right\}^{1 / 2}
$$

where $M$ is the mass of the parent particle and $m, \mu$ are the masises 
of the decay baryon and meson, respectively. In Table $\mathrm{V}$ we list widths calculated using the masses given by Eq. (3.9) for the case $R=8$. These values hould be regarded as lower limits, both because they increase with the scale of charmed particle masses, and also because we have neglected other channels which may be open. We have also listed branching ratios calculated with $R=8$ and in the limit of very large $R$ where mass differences within an SU(3) multiplet become negligible. However if baryon masses are really very high, the decay widths may become so broad that the resonance structure will completely disappear.

Strong baryon decay followed by the weak decay of a charmed meson will lead to a minimum of three particles in the final state as indicated

in Table V. Whatever the primary decay mode, since one weak transition is necessarily involved, the overall decay chain will satisfy the selection rules (4.12) and (4.13). If the widths of charmed baryons are sufficiently narrow, they will appear as resonances in multiparticle channels ( $\mathrm{n} \geq 3$ ) with "exotic" quantum numbers:

$$
\begin{array}{lc}
S=-1, Q=0,+1,+2 & \left(C_{0}, C_{1}\right) \\
S=-2, Q=0,+1 & (S, A) \\
S=-3, Q=0 & (T)
\end{array}
$$

for $C=+1$ states. States with $C=+2$ will decay into channels with multiplicity $\mathrm{n} \geq 5$ and quantum numbers:

$$
\begin{array}{ll}
S=-1, Q=+1,+2 & \left(x_{u, d}\right) \\
S=-2, Q=+1 & \left(x_{s}\right)
\end{array}
$$

In addition to decays of the type (4.14), there may be decays involving charmed or uncharmed vector mesons as well as spin $3 / 2$ baryons, for example:

$$
\begin{aligned}
& C^{++} \rightarrow \Delta^{++}+D^{0} \\
& T^{0} \rightarrow \Omega^{-}+\dot{F}^{+}
\end{aligned}
$$

Of course there is also a predicted spectrum of charmed baryons with spin $3 / 2$, whose properties we have' not discussed. The lowest of such states may be expected to form a $20^{\prime}$ of SU(4) characterized by a tetrahedral structure.

\subsection{Vector Meson Decays.}

r. In contrast to the case for baryons, the mass formulae of Sec. 3 indicate that vector mesons may have very narrow decay widths and will perhaps be stable against strong decays. Using the empirical relation:

$$
m_{k}^{2}-m_{\pi}^{2} \simeq m_{k}^{2} \text {. }-m_{p}^{2}
$$

we obtain from Eqs. (3.1) and (3.8): $m_{F \Delta}-m_{F} \leqslant m_{D}^{\Delta}-m_{D} \simeq\left(m_{P}^{2}-m_{\pi}^{2}\right) /\left(m_{D}+m_{D}\right) \lesssim 200 M_{e V}$
$m_{F \Delta}-m_{D} \simeq\left(m_{K}^{2}-m_{\pi}^{2}\right) /\left(m_{F}+m_{D}\right) \leqslant 250 M_{e V}$ The decay $\mathrm{F}^{*} \rightarrow \mathrm{F}+\pi$ is forbidden by isospin, and these results indicate that the decays

$$
F \rightarrow\left\{\begin{array}{l}
F+\eta \\
D+K
\end{array}\right.
$$

will not be energetically possible. The $F^{*}$ may decay weakly, for example:

$$
F+\rightarrow\left\{\begin{array}{l}
\dot{\hat{K}^{0}} K^{+} \\
\mu^{+} \nu
\end{array}\right.
$$


and the decay $\mathrm{F}^{*} \rightarrow \mathrm{F}+\mathrm{Y}$ is also possible, and is expected to be dominant. 13

The decay

$$
D^{*} \rightarrow \mathrm{D}+\pi
$$

may be allowed. We can estimate the decay width by comparing the available phase space with that for $\mathrm{K}^{*^{*}} \rightarrow \mathrm{K} \pi$, since the group structure is identical under the substitution $c \rightarrow s$. Neglecting the squared pion mass we have:

$$
\Gamma\{\mathrm{V}(\mathrm{M}) \rightarrow \mathrm{P}(\mathrm{m})+\pi\} \sim \frac{\mathrm{P}^{3}}{\mathrm{M}^{2}}=\frac{\left(\mathrm{M}^{2}-\mathrm{m}^{2}\right)^{3}}{\mathrm{M}^{5}}
$$

Then

$$
\Gamma\left(D^{*} \rightarrow \mathrm{D} \pi\right)=\left(\mathrm{m}_{\mathrm{K}} / \mathrm{m}_{\mathrm{D}^{*}}\right)^{5} \Gamma\left(\mathrm{K}^{*} \rightarrow \mathrm{K} \pi\right) \leq 3 \mathrm{MeV},
$$

for $R \geq 8$. 'If $R \geq 20, D^{*} \rightarrow D \pi$ is forbidden.

The state $\phi_{c}$ carries no charm and therefore can decay strongly into ordinary pseudoscalars. However we anticipate that $\phi_{C}$ is primarily a $\bar{c} c$ bound state, and its decay into uncharmed particles may be suppressed in the same way that the decay of $\phi \sim \bar{s}$ into non-strange particles is ,uppressed. According to Eqs. (3.6) and (3.8), the decays

$$
\varphi_{c} \rightarrow \bar{D} D, \bar{F} F
$$

will not be energetically possible, and the width of the $\phi_{c}$ may be comparable to the $\phi$ width. Very roughly we expect $(\Gamma \sim \mathrm{M})$

$$
\Gamma_{\varphi_{c}} \simeq \Gamma\left(\varphi_{c} \rightarrow \vec{k} k, 3 \pi, \ldots\right) \simeq \frac{m_{\varphi_{c}}}{m_{\varphi}} \Gamma(\psi \rightarrow 3 \pi, \eta \xi)
$$$$
\simeq 0.2\left(m_{\varphi_{c}} / m_{4}\right) \Gamma_{\varphi}^{\prime} \simeq 2 M_{e} v^{\prime}
$$

for $R=8\left(m_{\phi^{\prime}} \simeq 2\right)$. Furthermore, the suppression of the "favored" decay modes will enhance the leptonic branching ratio. The vector meson coupling to the photon is proportional to the charge of the bound quarks :

$$
g_{1 \varphi_{c}}=-2 g_{\gamma \varphi}
$$

\section{Thus we expect:} $\Gamma\left(\psi_{c} \rightarrow l^{+} l^{-}\right) \simeq 4\left(m_{\varphi_{c}} / m_{4}\right) \Gamma\left(\varphi \rightarrow l^{+} l^{-}\right) \simeq 4\left(m_{\psi_{c}} / m_{p}\right)\left(5 \times 10^{-1}\right) \Gamma_{\psi}$.

If the hadronic decay modes of the $\phi_{c}$. are suppressed as much as indicat ed in Eq. (4.16), we obtain for the lepton branching ratio

$$
\frac{\Gamma\left(\varphi_{e} \rightarrow l^{+} l^{-}\right)}{\Gamma_{\varphi_{c}}} \simeq 1 \%
$$




\section{-40- FE RMILAB-PUb-74/86-THY}

TABLE IV.

Branching Ratios of Nonleptonic Decays

of a Charmed $0^{-}$Meson

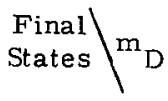

$\overline{\mathrm{K}} \pi$

$\overline{\mathrm{K}} 2 \pi$

$\overline{\mathrm{K}} 3 \pi$

$\bar{K} 4 \pi$

$\overline{\mathrm{K}} 5 \pi$

$\overline{\mathrm{K}} 6 \pi$

$\overline{\mathrm{K}} 7 \pi$

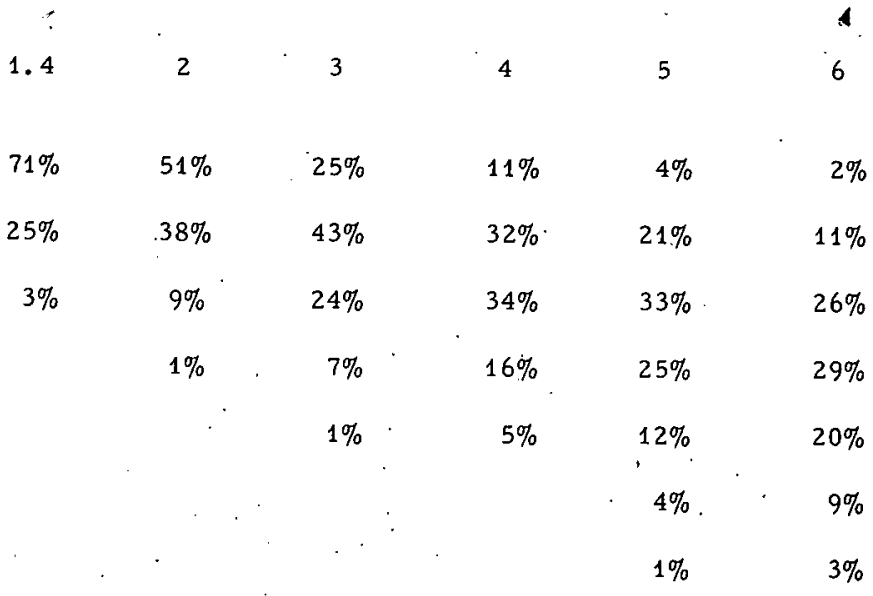

$-41-$

FE RMI LAB-Pub-74/86-THY

TABLE V.

Strong Decays of Charmed Baryons:

$$
\mathrm{B}^{\mathrm{C}} \rightarrow \mathrm{B}+\mathrm{P}^{\mathrm{C}} \text {. }
$$

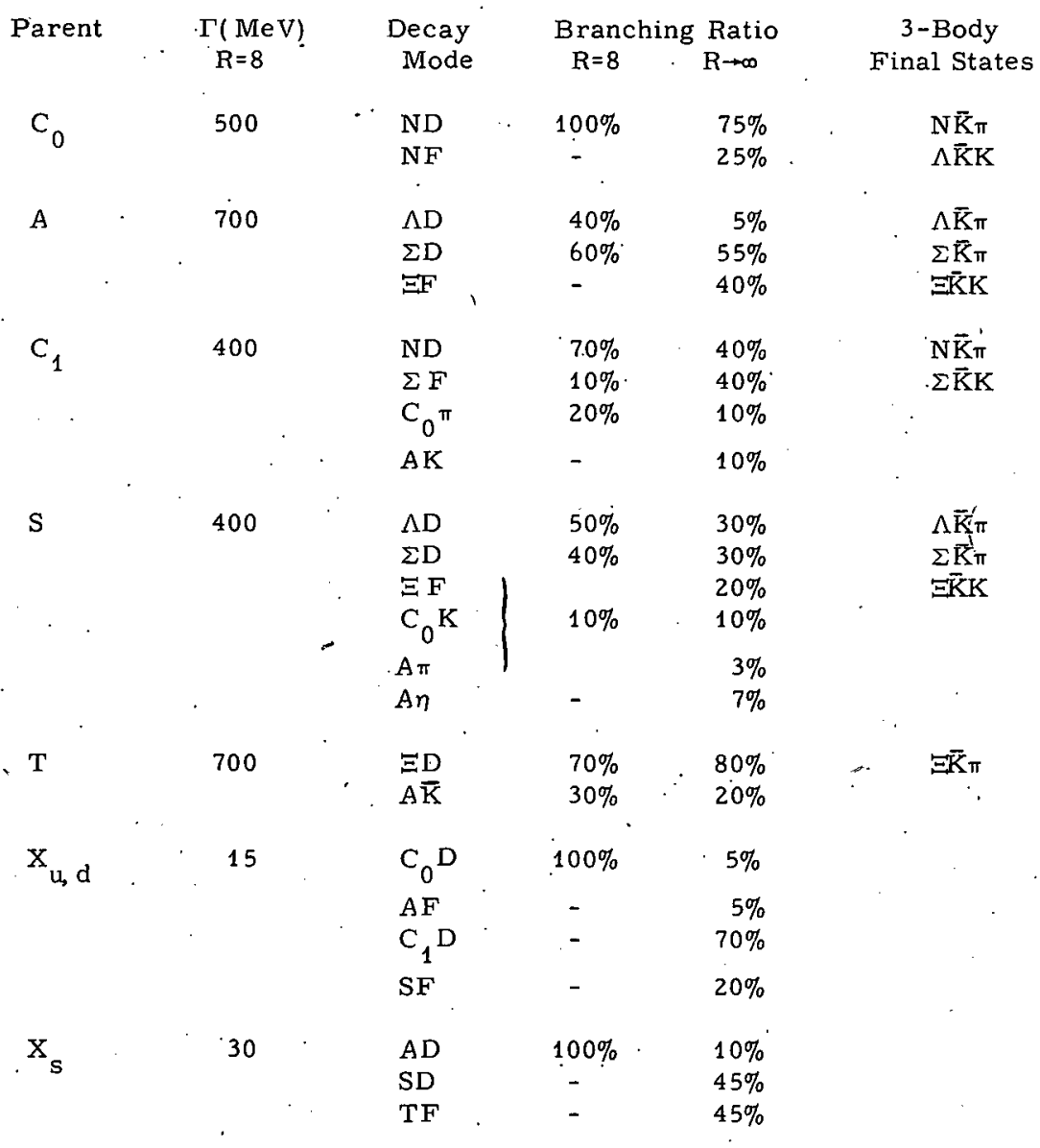




\section{PRODUCTION OF CHARMED PARTICLES}

\subsection{Diffractive Photoproduction of $\phi$}

- We have argued that $\phi_{\mathrm{c}}$ is mostly $\mathrm{c} \overline{\mathrm{c}}$ in the same way that $\phi$ is mostly ss. It has been pointed out by Carlson and Freund (1972) that $\phi_{c}$ might be produced diffractively by photons. In this subsection, we give a crude estimate for the diffractive production cross section of $\phi_{c}$ based on the vector meson dominance and the simple quark model.

In the simple quark model we have been using, the photon vector meson couplings, $g_{v}$, defined as

$$
\begin{aligned}
& \left\langle v\left|J_{\mu}^{e \cdot n}\right| 0\right\rangle=\frac{m_{v}^{2}}{g_{v}} \\
& \text { are in the ratio } \\
& \frac{g_{\rho}^{2}}{4 \pi}: \frac{g_{\omega}^{2}}{4 \pi}: \frac{g_{\varphi}^{2}}{4 \pi}: \frac{g_{\varphi c}^{2}}{4 \pi}=\frac{1}{9}: 1: \frac{1}{2}: \frac{1}{8}
\end{aligned}
$$

According to the usual lore of vector meson dominance, one has

$$
\frac{d \sigma}{d t}\left(f N \rightarrow \varphi_{c} N\right)=\frac{e^{2}}{g_{\varphi_{c}^{2}}^{2}} \cdot \frac{d \sigma}{d t}\left(\varphi_{c} N \rightarrow \varphi_{c} N\right)
$$

In the forward direction, the elastic differential cross section for $\mathrm{VN} \rightarrow \mathrm{VN}$ can probably be estimated fairly accurately from the optical theorem using the quark model estimate of the total cross section for VN. The total cross section for $\phi_{c} \sim c \bar{c}$ off a nucleon should be.the the same as for $\phi \sim s \bar{s}$ at sufficiently high energies where the mass difference between $s$ and $c$ may be neglected. Thus the simple quark model gives, with the additivity assumption,

$$
\dot{\sigma}_{T}\left(\phi_{C} N\right)=\sigma_{T}(\phi N)=\sigma_{T}\left(K^{+} N\right)+\sigma_{T}\left(K^{-} N\right)-\sigma_{T}\left(\pi^{-} N\right) .
$$

The lașt equality was recentiy studied and parametrized by Lipkin, ${ }^{14}$ who gave

$$
\sigma_{T}\left(\phi_{C} N\right)=\sigma_{T}(\phi N)
$$

$=13.5+1.25 \ln (\mathrm{p} / 20 \mathrm{GeV}) \mathrm{mb}$

where $p$ is the laboratory momentum.

By the optical theorem

$$
\left.\frac{d \sigma}{d t}\right|_{t=0} \simeq \frac{1}{16 \pi} \sigma_{r}^{2}
$$

Equation, (5.3) implies

$$
\begin{aligned}
& \left.\frac{d \sigma}{d t}\left(y \dot{\prime} \rightarrow \psi_{c} N^{\prime}\right)\right|_{t=0} \simeq \alpha\left(\frac{4 \pi}{g_{0}^{2}}\right) \frac{\delta}{9} \cdot \frac{1}{16 \pi} \\
& \times\left[13.5+1.25 l_{n}(p / 20 G+V)\right]^{2} m b \frac{1}{0.357(G \ldots i)}
\end{aligned}
$$

where use has been made of Eq. (5.2). Thus, at $p=200 \mathrm{GeV}$, one expects

$$
\left.\frac{d \sigma}{d t}\right|_{t=0}\left(\gamma N \rightarrow \varphi_{c} N\right) \simeq 40 \mu b /\left(G_{e} \cdot v\right)^{2}
$$

If indeed $\phi_{\mathrm{c}}$ is produced as copiously as $\phi$ in a photoproduction 
experiment, we expect the signal in the muon pair channel to be $\sim 10^{2}$ times stronger for the former than for the latter, because, as discussed in. Sec. 4.4 , the branching ratio for $\phi_{c} \rightarrow \mu \vec{\mu}$ is probably larger by about this factor than that for $\phi \rightarrow \mu \bar{\mu}$, if $\phi_{C}$ is stable against decay into two charmed pseudoscalar mesóns. As remarked by Carlson and Freund (1972), existing data on the photoproduction of $\mu$-pair's suggest already that $\operatorname{M}\left(\phi_{c}\right)>2 \mathrm{GeV}$ (see Hayes, et al. , 1970).

Similar considerations apply to leptoproduction of charmed vector bosons and $\phi_{c}$. Thus, $\phi_{c}$ may be produced diffractively in electron-, muon-, and neutrino (neutral current)-scattering experiments, while charged current effects in neutrino experiments may include diffractive production of $\mathrm{F}^{* \pm}$ :

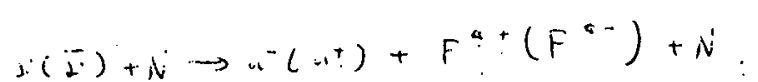

\subsection{Neutrino Reactions}

\subsubsection{Quasi-elastic Scattering.}

Near the threshold for charmed particle production one might expect the dominant process to be the production of a single baryon state. However, this process is suppressed by the $\Delta S=\Delta C$ selection rule and occurs at a level of $\sin ^{2} \theta . c$ The allowed "elastic" reactions are:

$$
\begin{aligned}
& \dot{L}+\mathrm{p} \rightarrow \mathrm{C}_{1+{ }^{+}+\mathrm{h}^{-}}^{+} \\
& \dot{\nu}+n \rightarrow\left\{\begin{array}{l}
\mathrm{C}_{1}^{+}+\mathrm{H}^{-} \\
\mathrm{C}^{+}+\mathrm{C}^{-}
\end{array}\right.
\end{aligned}
$$

Neglecting form factors and weak magnetism, the hadronic matrix element is of the form

$$
\left\langle c\left|J_{\mu}\right| N\right\rangle=\sin \theta_{c} \vec{u}_{e} f_{\mu}\left(g_{v}-g_{A} \gamma_{5}\right) u_{N}
$$

and the differential cross section is:

$$
\begin{aligned}
\frac{d \sigma}{d y}= & \frac{2}{\pi} G_{F} m_{N}^{2} \sin ^{2} \theta_{c} \frac{1}{y}\left\{\left(\frac{g_{A}+g_{V}}{2}\right)^{2}\left(1-y_{t h}\right)\right. \\
& \left.\left.+\frac{\left(g_{A}-g_{V}\right.}{2}\right)^{2}(1-y)\left(1-y^{2}+y_{t h}\right)\right\},
\end{aligned}
$$

where $\mathrm{y}$ is the fraction of the incident neutrino energy transmitted to the baryon:

$$
\dot{y}=E_{C} / \tilde{t}_{\nu}
$$

The threshold conditions for production of a charmed state are:

$$
\begin{aligned}
& E \geqslant E_{t h} \simeq m_{c}^{i} / 2 m_{N}, \\
& y \geqslant y_{t h}=E_{t h} / E_{\nu} .
\end{aligned}
$$

In Fig. 4 we show the differential cross section for production of a spin $1 / 2$ state for pure $V-A\left(g_{V}=g_{A}\right)$ and pure $V+A\left(g_{V}=-g_{A}\right)$ couplings, assuming $\mathrm{m}_{\mathrm{c}}=3 \mathrm{GeV}, \dot{\mathrm{E}}_{\nu}=25 \mathrm{GeV}$. In either case the effect is less than a percent of the total measured cross section which we approximate by the scaling form $\left(\sigma \equiv \sigma^{\mathrm{n}}+\sigma^{\nu \mathrm{p}}\right)$ :

$$
\frac{d \sigma^{2}}{d y}{ }_{\text {rotal }}^{2} \simeq \frac{G_{F}^{2}}{\pi} m_{N} E_{\nu} .
$$


The couplings for the reactions (5.7) may be determined in the SU(4) limit from the known hyperon decay parameters. We then obtain a mixture of $\mathrm{V}-\mathrm{A}$ and $\mathrm{V}+\mathrm{A}$ contributions to the cross section as indicated in Fig. 4. The production cross section for $\mathrm{C}_{0}^{+}$is the more favorable, as it is nearly pure V-A. If the mass of this state is as low as, say 2. $5 \mathrm{GeV}$, it could be produced at CERN and Brookhaven energies.

A1though the distribution of Fig. 4 shows a sharp fall off with $y$ for fixed neutrino energy, if one averages the y distribution over a range of energies near threshold, the net effect will be an increase with $y$ since lower energies may contribute for higher $y$.

The decay of the charmed particle satisfies (to order $\sin ^{2} \theta_{c}$ ) $\Delta C=\Delta S=-i$. Therefore, the production of a $C=+1, S=0$ state by low energy neutrinos $\left(E_{\nu}<10 \mathrm{GeV}\right.$ with $\left.\left\langle\mathrm{E}_{\nu}\right\rangle=2 \mathrm{GeV}\right)$ would lead to the appearance of $S=-1$ final states, increasing sharply with $E_{\nu}$ and $y$ above the threshold values $\left(E_{t h} \simeq 3 \mathrm{GeV}, \mathrm{y}_{\mathrm{th}} \simeq 0.3\right.$ for $\left.\mathrm{m}_{\mathrm{c}} \simeq 2.5 \mathrm{GeV}\right)$. However the absolute value of the cross section remains very small $\left(\sigma_{c} / \sigma_{\text {tot }}<1 \%\right)$ and charm production is likely to be masked by associated production of strange particles where one strange particle escapes detection. Since at low energies associated production may also show threshold effects, ${ }^{15}$ the detection of charm will be very difficult unless the leptonic branching ratio is significant.

\section{2.2 Diffractive Production of Vector Mesons}

A more copious source of charmed particle production may be through the diffractive process discussed in Sec. 5.1. The $F^{*}$ couples to the weak current with amplitude proportional to $\cos \theta_{c}$. If its leptonic branching ratio is appreciable, it will provide a source of dileptons. The electroproduction of vector mesons is not well understood (Gettfried, 1971), but we might guess that

$$
\frac{\sigma\left(\nu+N \rightarrow \mu+F^{*}+N\right)}{\sigma(\nu+N \rightarrow \mu+\text { any thing })} \sim \frac{\sigma\left(e+p \rightarrow e+\rho^{a j} \omega+p\right)}{\sigma(e+p \rightarrow e+\text { anything })} .
$$

The right-hand side (Berkelman, 1971) is about $10 \%$ for $\left|Q^{2}\right| \lesssim$ 1. $2(\mathrm{GeV} / \mathrm{c})$. Similar estimates should obtain for $\phi_{\mathrm{c}}$ production by neutral current couplings. Decays of $F^{*}$ are discussed in Sec. 4. 4.

\subsubsection{Deep Inelastic Production}

For neutrino energies above charm threshold, it is probably reasonable to apply the quark parton model which appears to describe well deep inelastic production of ordinary hadrons. Estimates of charmed. particle production can then be deduced from cross sections for elementary $v$-quark scattering (Altarelli, et al., 1974; Gaillard, 1974):

$$
\begin{array}{ll}
\nu+d \rightarrow c+\mu^{-} & \sigma-\sin ^{2} \theta_{c} \\
\nu+s \rightarrow c+\mu^{-} & \sigma=\cos ^{2} E_{c}
\end{array}
$$

and the charge conjugate processes. The crossed processes (e.g., $\bar{v}+\mathrm{c} \rightarrow \mu^{+}+s$ ) could also occur if charmed partons are present in the nucleon. The total cross section for charmed particle production depends 
on the distribution of partons within the nucleon. Comparison of

electroproduction and neutrino data suggests that the nucleon consists primarily of valence $u$ and $d$ partons. If $F_{i}$ is defined as the integrated distribution function for the i-type parton in the proton, the data yield the following constraints (Altarelli, et al., 1974; Gaillard, 1974)

$$
\begin{aligned}
& F_{\bar{u}}+F_{\bar{j}}=(0.05 \pm 0.02)\left(F_{u}+F_{d}\right), \\
& F_{s}+F_{j} \leq(0.25)\left(F_{u}+F_{d}\right),
\end{aligned}
$$

and if charmed partons are included:

$$
F_{c}+F_{\bar{c}} \dot{s}(0.06)\left(F_{u}+F_{d}\right) \text {. }
$$

The distribution functions are positive definite; for the purpose of discussion we shall assume:

$$
\begin{aligned}
& F_{c} \simeq F_{\bar{c}} \simeq 0, \\
& F_{s} \simeq F_{\bar{s}} \doteq 0.1\left(F_{i}+F_{d}\right) .
\end{aligned}
$$

However, it is unlikely that the strange quark content is as high as this upper limit, since the $\bar{u}$ and $\bar{d}$ content is much smaller.

The contribution of an elementary $\nu$-parton scattering process to the total cross section is proportional to the elementary scattering cross section and to the distribution function of the parton. Using the reļations :

$$
\sigma(\bar{\nu}+q)=\sigma(\nu+\bar{q})=\frac{1}{3} \sigma(\nu+q)=\frac{1}{3} \sigma(\bar{\nu}+\bar{q}),
$$

we may estimate the relative cross section for charm production.

Distribution functions for the neutron are related to these for the proton by charge symmetry: $\mathrm{u} \longrightarrow \mathrm{d}$ for' $\mathrm{n} \leftrightarrow \mathrm{p}$. Neglecting contributions of order' $\sin ^{2} \theta_{c}$, we obtain for neutrino scattering from a heavy nucleus $(A=2 Z)$

$$
\begin{aligned}
& \frac{\sigma_{c}^{\nu}}{\sigma_{t u t_{\alpha} !}^{\nu}} \approx \frac{2 F_{s}}{\left[F_{u}+F_{d}+\frac{1}{3}\left(F_{\bar{u}}+F_{\bar{\alpha}}\right)+2 F_{s}\right]} \leqslant 16 \% \\
& \frac{\sigma_{c}^{\bar{\nu}}}{\sigma_{T_{0}+a l}^{\bar{z}}} \simeq \frac{2 F_{s}}{\left[F_{\bar{u}}+F_{\bar{d}}+\frac{1}{3}\left(F_{u}+F_{d}\right)+2 F_{j}\right]} \leqslant 35 \%
\end{aligned}
$$

However, these bounds can be reached only asymptotically. Even for relatively high neutrino energies, thresholds may be important in limiting the allowed region of phase space. We define the usual scaling variables (see Fig. 5):

$$
\begin{aligned}
& x=-i^{2} / 2 \nu \\
& y=\nu / m_{N} E_{\nu}
\end{aligned}
$$

where $q$ is the momentum transfer and $v=p \cdot q=m_{N}\left(E-E^{\prime}\right)$. In general $\mathrm{x}$ and $\mathrm{y}$ satisf $\mathrm{y}$

$$
0 \leq x, y \leq 1
$$

For production of a state with mass $\mathrm{m}_{\mathrm{c}}$, the allowed phase space (deRujula, et al. , 1974) is limited to: 


$$
\begin{aligned}
& \frac{E_{t h}}{E_{\nu}} \leq y \leq 1, \\
& 0 \leq x \leq 1-\frac{E_{+h}}{y E_{\nu}}
\end{aligned}
$$

where $E_{t h} \simeq m_{c}^{2} / 2 m_{N}$ is the threshold energy. If the lowest charmed state has a mass of, say, $3 \mathrm{GeV}$, we might expect the parton model to become relevant at a slightly higher hadronic mass, say, $5 \mathrm{GeV}$. Then for $\mathrm{E}_{\nu}=25 \mathrm{GeV}$, the parton model would be applicable in the region:

$$
0 \leqslant x \leqslant 0.5 \quad, \quad 0.5 \leqslant y \leqslant 1 .
$$

Under these assumptions the contribution of the scattering process $(5.12)$ to the differential cross section takes the form $\left(\sigma \equiv \sigma^{\text {nn }}+\sigma^{\text {pp }}\right.$ )

$$
\frac{d \sigma^{\nu(i)}}{d x d y}=\frac{4 G_{F}^{2} m_{N} E}{\pi} f_{S(s)}(x) \Theta\left(w^{2}-m_{c}^{2}\right)
$$

where $f(x)$ is the parton distribution function:

$$
F_{i}=\int_{0}^{1} d x f_{i}(x)
$$

$m_{c}=5 \mathrm{GeV}$ is the "scaling threshold" mass and $W$ is the total hadronic invariant mass:

$$
w^{2}=2 m_{N} E_{2} y(i-x)
$$

In Figs. 6 and 7 we show the contribution of the cross section $(5.29)$ to the $\mathrm{y}$ and $\mathrm{w}^{2}$ distributions (shaded areas); we have assumed the parametrization: $:^{16}$

$$
f_{s}=f_{\bar{B}}=0.2(1-x)^{7}
$$

which corresponds to

$$
2 F_{s}=0.05=0.1\left(F_{u}+F_{d}\right) \text {. }
$$

If the strange particle content of the nucleon is important, the onset of charmed particle production is expected to be more pronounced in anti-neutrino events than in neutrino events. This is because both $v-s$ and $\bar{v}-\bar{s}$ scatterings are isotropic, giving a flat $\mathrm{y}$ distribution as for $\nu$-d.scattering, while the dominant contribution for anti-neutrino scattering:

$$
d \sigma\left(j+u \rightarrow \mu^{+}+d\right) \approx(1-g)^{2}
$$


falls off at high $y$ where charmed particle production will first appear. In Fig. 6, the dashed line shows the expected y-distribution for $\bar{\nu}$ scattering in the absence of charm (assuming a 5 percent $\overline{\mathrm{u}}+\overline{\mathrm{d}}$ content). the solid line shows the full distribution assuming the above parameters. Figure 7 shows the invariant mass distributions where we have further. assumed simple parametrizations for the remaining structure functions:

$$
\begin{aligned}
-f_{N} & \equiv f_{u}+f_{d}=2(1-x)_{1}^{3} . \\
f_{N} & \equiv f_{\bar{u}}+f_{\bar{d}}=0.2(1-x)^{7}
\end{aligned}
$$

In fact, the $(1-x)^{3}$ behavior for $f_{N}$ is valid only near $x=1$; we must have isee, e.g., Llewellyn-Smith, 1973):

$$
f_{N} \rightarrow f_{i} \text { for }: x \rightarrow 0 \text {. }
$$

Therefore the contribution from $\mathrm{f}_{\mathrm{N}}$ falls off more rapidly with increasing $W^{2}$ than indicated in Fig. 7 ; this will further enhance the effect of charmed particle production. Of course, we have neglected "pre-scaling" contributions to charm production; their effect will certainly be to smooth out the threshold effects shown in Figs. 6 and 7.

The above discussion rests entirely on the assumption that there is a significant strange parton content in the nucleon. If this is the case, since both the production and decay satisfy $\Delta S=\Delta C$, there will be no net change of strangeness. The production of charm will appear as associated production of strange particles; possibly accompanied by a dilepton in the final state.

If there is no appreciable strange parton content, charmed particles will be produced at a-level of $\sin ^{2} \theta_{c} \simeq 4$ percent via the $\nu$-parton scattering process of Eq. (5.11). Since strangeness is conserved in the production, the, net change of strangeness will satisfy

$$
\Delta S=-\Delta Q
$$

for non-leptonic decays of the charmed particles. Using parton model estimates, we obtain for the relative cross sections

$$
\begin{aligned}
& \sigma_{c}^{\nu} / \sigma_{\text {total }}^{\nu} \simeq \tan ^{2} \theta_{c} \simeq 4 \% \\
& \sigma_{c}^{\bar{\nu}} / \sigma_{\text {total }}^{\bar{\nu}} \simeq 0 . \varsigma^{-\eta_{0}}
\end{aligned}
$$

The extra suppresion in the anti-neutrino case is due to the fact that the scattering must occur from $\vec{d}$. For ordinary $\Delta S=\Delta Q$ transitions the situation is reversed:

$$
\begin{aligned}
\sigma_{s}^{\nu} / \sigma_{r_{0} t a l}^{\nu} & \simeq 0.5-1 \% \\
\sigma_{s}=1 \sigma_{\text {rotal }}^{\nu} & \simeq 4 \% \%
\end{aligned}
$$

thus at energies which are asymptotic with respect to the charm threshold one expects to see a predominance of $S=-1$ states at a level of about 4 percent in both neutrino and anti-neutrino events. In neutrino events they may be accompanied by a di-lepton associated with the leptonic decay of a charmed particle.

\section{3 e- Annihilation}

Once the energies of electron-positron colliding beams are high ènough, pair production of charmed particles, and resonant production of $\phi_{C}$ are expected to proceed without inhibition. 
The process $\mathrm{e}+\overline{\mathrm{e}} \rightarrow \phi_{\mathrm{c}}$ should be very similar to $\mathrm{e}+\overline{\mathrm{e}} \rightarrow \phi$; in particular, $\mathrm{e}+\overline{\mathrm{e}} \rightarrow \phi_{\mathrm{c}} \rightarrow \mu+\bar{\mu}$ presents a clean way of measuring the mass and width of the $\phi_{c}$ meson.

The processes $\mathrm{e}+\overline{\mathrm{e}} \rightarrow \mathrm{D}+\overline{\mathrm{D}}+$ pions, or $\mathrm{F}+\overline{\mathrm{F}}+$ pions, or $\mathrm{D}+\overline{\mathrm{F}}+$ pions and kaons, or its charge conjugate reaction should occur copiously above threshold. An interesting reaction is

$$
\begin{array}{r}
e+\bar{e} \rightarrow D^{0}+\stackrel{\bar{D}^{0}}{L^{+}} K^{+}+\pi^{-} \\
\longrightarrow K^{-}+\pi^{+}
\end{array}
$$

which will tell us about the mass of the D mesons unambiguously.

[As pointed out by H. Lipkin (private communication), the process $e^{+}+e^{-} \rightarrow D^{0}+\bar{D}^{0}$ is forbidden in the exact SU(4) limit. $]$ Another signature of charm pair production is the observation of single $\mu^{\prime} s$ in coincidence with strange particles: these events can arise from one of the pair decaying leptonically and the other nonleptonically, i e. ,

$$
\begin{aligned}
e+\bar{e} & \rightarrow D^{+}+D^{-}+\cdots \\
& \rightarrow \mu^{-}+\cdots(\text { (eptonic) } \\
& \rightarrow \dot{K}+\cdots \text { ( hadronic) }
\end{aligned}
$$

All these final states should occur in principle also in p $\bar{p}$ annihilation.

Finally, a remark is in order on the ratio $R$;

$$
R=\frac{\sigma(e+\bar{e} \rightarrow \text { hadirons })}{\sigma(e+\bar{e} \rightarrow \mu+\bar{\mu})} \text {, }
$$

which is found to be in the neighborhood of 5 at the current SPEAR energies $<5 \mathrm{GeV}$. In the three-color quark model which is asymptotically free, the asymptotic behavior of $\mathrm{R}$ is given by ${ }^{17}$

$$
R=2\left(1+\frac{c_{3}}{\ln s / \mu^{2}}\right), \quad c_{3}=4 / 9
$$

for the quarks of charges $2 / 3,-1 / 3$ and $-1 / 3$;

$$
R=\frac{10}{3}\left(1+\frac{C_{4}}{\ln 5 / 4^{2}}\right) ; \quad C_{4}=\frac{12}{25}
$$

for four quarks of charges $2 / 3,2 / 3,-1 / 3$ and $-1 / 3$. We note that the approach to the asymptotic value $(R=2$ or $10 / 3)$ is from above. It is tempting to conjecture (which can be disproved soon) that (1) we are in the regime where the asymptotic forms above are valid, and (2) the currently large value of $R$ is in fact associated with the onset of charm production, and therefore with the transition from one asymptotic form to the other. In Fig. 8 , we plot experimental values for $R$ with the curves of Eqs. (5.22, 5.23), assuming, arbitrarily, $\mu=2 \mathrm{GeV}$. The following remarks were made to us by $\mathrm{H}$. Lipkin, and we shall include them here with Professor Lipkin's kind permission.

\section{The large charge of the charmed quark leads to a large} predicted cross section for the production of charmed particle pairs once threshold effects are no longer relevant. Standard quark parton 
-arguments would suggest that at sufficiently high energy $40 \%$ of all. events should contain charmed particle pairs.

2. The dominant decay mode of charmed particles is nonleptonic with a strange particle in the final state. This implies that at sufficiently high energies roughly half of all events should contain strange particles in the final state. This is to be contrasted with prediction of the quark parton model for the case where there are no charmed particles which gives strange particles present in only $1 / 6$ of the events.

3. The nonleptonic decay of a charmed particle into nonstrange particles is suppressed by a factor $\sin ^{2} \theta$ where $\theta$ is the Cabibbo angle. However, if one of a pair of charmed particles decays in the nonstrange mode while the other decays into strange particles there will be an apparent violation of strangeness conservation in the final state which will contain only a single strange particle. If the probability of producing a charmed pair is $40 \%$ as given by the quark parton model and either one of the pair has a probability $\sin ^{2} \theta$ of decaying into nonstrange particles the probability of observing strangeness violation in a given event is

$$
P(\Delta S=1)=0.8 \sin ^{2} \theta
$$

This is by no means a small probability. Thus if the colliding beam experiments show a large number of events containing strange particles approaching the order of $50 \%$ this can be taken as an indication that charmed particles are indeed produced. It would then be worthwhile to make a special effort to examine the events in which strange particles are produced very carefully to note whether there are any cases in which one and only one strange particle is produced. This would involve finding events in which all particles are detected and a positive identification can be made of charge particles as either pion or kaon. Since this may be difficult with the kind of detectors , used at SPEAR it could be left as a second stage in the charmed particle search. The first stage would be to establish the probability of strange particle production to determine whether there is any anomalous production suggesting the existence of charmed particles.

\section{4 Lepton Production at High Momentum Transfer}

The leptonic decays of charmed particles can provide a source of direct leptons in hadronic collisions. The expected rates, of course, depend on two unknown factors -- charm production rates and the branching ratios for leptonic decays -- but one can try to make some educated guesses.

Consider the chain 


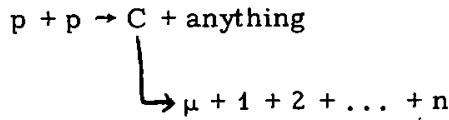

where $\mathrm{C}$ is a charmed particle with large $\dot{\mathrm{p}}_{\perp}$ and $1,2, \ldots, \mathrm{n}$ stand for decay products of $\mathrm{C}$ other than the observed $\mu$. Then the $\mu$-distribution

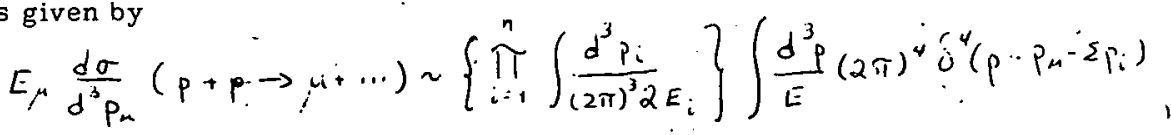

$$
\begin{aligned}
& \times \frac{1}{2 M \Gamma}\left|T\left(p ; p_{\mu}, p_{1}, \cdots, p_{r}\right)\right|^{2} E \frac{d \sigma}{d^{3} p}(p+p \rightarrow C+\cdots),
\end{aligned}
$$

where $M$ and $\Gamma$ are the mass and decay width of $C$ and $T$ is the decay amplitude for $C \rightarrow \mu+1+2+\ldots+n$. If we assume a distribution of the form ${ }^{18}\left(p_{1}>2 \mathrm{GeV}\right)$

$$
E \frac{d \sigma}{d^{3} p}(p+p \rightarrow C+\cdots) \sim p_{i}^{-8.2} \exp \left(-26.1 p_{\perp} / \sqrt{5}\right) \text {, }
$$

then $E_{\mu} d \sigma / d^{3} p_{\mu}$ will have a similar shape. The reduction factor $r$, defined as

$$
\Omega=E_{\mu} \frac{d \sigma}{d^{3} p_{n}}(p+p \rightarrow \mu+\cdots) / E \frac{d \sigma}{d^{3} p}(p+p \rightarrow C+\cdots) \times\left. B\right|_{\theta=90^{\circ}},
$$

where ${ }^{*} B$ is the branching ratio into the channel $C \rightarrow \mu+1+2 \ldots+n$, is about $10^{-1}$ for a two-body decay ${ }^{19}$ (such as $\mathrm{C} \rightarrow \mu \bar{\mu}, \mu \nu$ ). For a three body decay ${ }^{19}$ such as $D \rightarrow K \ell v, r$ depends on the $K-D$ mass ratio and on $p_{1}$ (see Fig. 9). In the case when the mass of the parent particle is comparable to the observed $p_{1}$, one expects important contributions from charmed particles produced at rest or with small $p_{1}$, where the distribution (5.24) is no longer valid. The leptons from this source $\left(\left(p_{1}\right)_{\ell}<m / 2\right)$ will have a different distribution. Without a precise model for the production distribution, it is difficult to estimate the lepton yield, which is expected to depend sensitively on the laboratory angle.

At energies which are sufficiently high that mass differences become unimportant, we expect charmed particle to be comparable to strange particle production. At Fermilab energies and for transverse momentum:

$$
2 \mathrm{GeV}<\mathrm{p}_{\mathrm{T}}<6 \mathrm{GeV}
$$

the observed $\mathrm{K}$ to $\pi$ production ratios are (Cronin, et al. , 1973)

$$
\begin{aligned}
& \mathrm{K}^{+} / \pi^{+} \simeq 0.5 \\
& \mathrm{~K}^{-} / \pi^{-} \simeq 0.2
\end{aligned}
$$

although there is some variation with energy and transverse momentum. Since the initial state carries neither charm nor strange:less, we expect. the final state to be invariant under the substitution $s \rightarrow c$. Then we guess that in some asymptotic limit:

$$
\begin{aligned}
& \overline{D^{\circ}}(u \bar{c}) \simeq K^{+}(u \bar{s}) \\
& D^{0}(c \bar{u}) \simeq K^{-}(s \bar{u})
\end{aligned}
$$

Another reasonable guess is 
$F^{+}(c \bar{s}) / K^{r}(u \bar{s}) \simeq D^{\top}(\bar{c} \bar{d}) / \pi^{+}(u \bar{d})$.

$F^{-}(s \bar{c}) / K^{-}(s \bar{u}) \bumpeq D^{-}(d \ddot{c}) / \pi^{-1}(d \bar{u})$.

In the energy and $p_{\perp}$ regions considered here there is a slight preference for positive pions, but to a good approximation (Appel, et al. , 1974a; Cronin, et al: , 1973):

$$
\pi^{+}=\pi^{0}=\pi^{-}
$$

By analogy we assume:

$$
\begin{aligned}
& D^{\prime}=D^{\circ} \\
& D^{-}=\overline{D^{\circ}}
\end{aligned}
$$

Then we obtain for the predicted asymptotic ratios at, e.g., $p_{1}=3 \mathrm{GeV} / \mathrm{c}$,

$$
\begin{aligned}
& \bar{D} / \pi \equiv\left(D^{-}+\bar{D}^{5}\right) / \pi^{0} \simeq 1 \\
& D / \pi^{0} \equiv\left(D^{+}+D^{c}\right) / \pi^{0} \simeq 4.4 \\
& F^{-} / \pi^{\circ} \simeq F^{+} / \pi^{\circ} \simeq 0.1
\end{aligned}
$$

The decay mode which will give the largest yield of high $p_{\perp}$ leptons is the one with the lowest multiplicity. For the $\mathrm{D}$ mesion, the two body leptonic decay is suppressed by a factor of $\sin ^{2} \theta_{c}$ as well as by helicity conservation (see Sec. 4 ).

$$
\Gamma\left(D_{\mu_{2}}\right) / \Gamma\left(D_{2_{3}}\right)<2 \times 10^{-3}
$$

for $\mathrm{m}_{\mathrm{D}}>1.4$. However, the two-body decay of the $\mathrm{F}$. meson can be important if the mass is low:

$$
\Gamma\left(\dot{F}_{\mu_{2}}\right) / \Gamma\left(\Gamma_{l_{3}}\right) \simeq \begin{cases}0.05 & m_{F}=1.5 \mathrm{GeV} \\ 0.015 & m_{F}=2 \mathrm{G} \cdot \mathrm{v}\end{cases}
$$

Using the mass values $m_{D}=1.4, m_{F}=1.5$, and the production rates of Eq. (5.31), we obtain for the predicted yields of high $\mathrm{p}_{\mathrm{T}}$ leptons :

$$
\begin{aligned}
\mu^{-1} / \pi^{\circ} & =\left(1.7 \times 10^{-3}\right) B_{k} \\
l^{+}+/ \pi^{\circ} & \simeq\left(1.3 \times 10^{-3}\right) B_{l} \\
e^{-} / \pi^{\circ} & \simeq\left(0.6 \times 10^{-3}\right) B_{Q} \\
e^{+} / \pi^{\circ} & \simeq\left(0.3^{3} \times 10^{-3}\right) B_{l}
\end{aligned}
$$

where $B_{\ell}$ is the total leptonic branching ratio, assumed to be the same for all charmed pseudoscalars. The $\mu$-e asymmetry is due to the impor-

\section{tance of the $F \rightarrow \mu \nu$ mode. For high masses, two-body decays are}

negligible. For three-body decays, the suppression factor is reduced at high mass, but we also expect the branching ratio to decrease since channels such as

$$
\begin{aligned}
& D \rightarrow(3 K) l_{\nu} \\
& \cdot F \rightarrow(2 \nu) l_{\nu}
\end{aligned}
$$

will have reasonable phase space. The charge asymmetry may also be less pronounced than indicated by the above predictions. In the case of strange particles the process

$$
\mathrm{P}+\mathrm{N} \rightarrow \mathrm{N}+\mathrm{Y}+\mathrm{K}^{+}
$$

is energetically more favorable than $\mathrm{K}^{-}$pair production:

$$
\mathrm{p}+\mathrm{N} \rightarrow \mathrm{N}+\mathrm{N}+\mathrm{K}+\overline{\mathrm{K}}
$$

and, presumably this accounts for the observed $\mathrm{K} / \overline{\mathrm{K}}$ asymmetry. If charmed baryon masses are such that. (see Sec. 3): 


\section{$m_{C}>m_{N}+m_{D}$}

$D$-pair production may be relatively less suppressed. Furthermore, if charmed baryons are very heavy, their production at rest and subsequent decay could yield high $p_{\perp} \mathrm{D}^{+}$mesons (but these would have a different $p_{\perp}$ distribution). In any case, the yields quoted above should be regarded as upper limits, particularly for high masses.

Another potentially important source of leptons is the $\phi_{c} \cdot$ As it can be produced singly, it may be more abundant than charmed mesons. For leptons with $\mathrm{p}_{\perp} \doteq \mathrm{m}_{\phi_{\mathrm{c}}}$, we expect a yield:

$$
e^{5} / \pi^{\circ}=\mu^{ \pm} 1 \pi^{\circ}=\left(\varphi_{0} / \pi^{\circ}\right) \times 5 \times 10^{-4} .
$$

if the total leptonic branching ratio of $\phi_{\mathrm{c}}$ is $\sim 10^{-2}$. For massive $\phi_{\mathrm{c}}$ we expect a peak in the lepton distribution at $p_{\perp}=m_{\phi_{C}} / 2$.

Recent experiments at Fermilab and at the CERN ISR show unexpectedly large yields of leptons at high momentum transfer: ${ }^{22}$

$$
e \pm / \pi^{\circ}=4 \pm / \pi^{\circ} \sim 10^{*}
$$

There are no significant asymmetries and no observed threshold effects or structure such as might be expected in the case of very massive sources. If the observed signal has anything to do with charm, the most likely candidate is $\phi_{\mathrm{c}}$ with $\mathrm{m}_{\phi_{\mathrm{c}}} \lesssim 3$ or $4 \mathrm{GeV}$.

\section{5 Associated Production in Strong Interactions}

One should stress again the similarity of charm with strangeness: ordinary hadronic reactions can produce charmed particles in pairs.
Examples of such reactions, as mentioned before, ${ }^{8}$ are

$$
\pi^{-p} \rightarrow M_{c} B_{c}
$$

and

$$
\stackrel{p p \rightarrow \stackrel{N^{*}}{\prime} P^{\prime}}{\longrightarrow} M_{c} \stackrel{B_{c}}{L_{\longrightarrow} \rightarrow M_{c} B}
$$

The first reaction involves charm exchange. If charmed particles are fairly massive and their Regge trajectories are of the usual slope $\alpha^{\prime \prime} \simeq 1 \mathrm{GeV}^{-2}$, the intercepts of these trajectories may be fairly low. One'would thus expect the associated-production reaction to be most useful not too far above threshold. The "diffractive excitation" reaction $\mathrm{pp} \rightarrow \mathrm{N}$. $\mathrm{p} \rightarrow \ldots$ suffers from no such problem, and can be useful at any energy.

.




\section{DETECTION OF TRACKS OF CHARMED PARTICLES}

Sufficiently light charmed particles may be detected via their tracks, in emulsions, and (under extremely favorable circumstances). perhaps also in bubble chambers.

We have argued that the semileptonic decay of a charmed particle should lead to

$$
\Gamma_{s L} \approx\left(10^{\prime \prime} / \sec \right) \times\left[M\left(\operatorname{cov}^{\prime}\right)\right]^{5}
$$

and the total decay rate could be anywhere from twice to a hundred times this value. Let us assume for the present that

$$
\Gamma_{\text {Tot }=1}=10^{12} \times[M(\operatorname{Cr} l)]^{5} \operatorname{sic}^{-1}
$$

realizing that this estimate could err by a factor of 10 in either direction.

Then the mean path length transversed by such a particle of laboratory momentum $\mathrm{p}$ is

$$
l=f_{c \tau}=300 \mu \times\left(p / M^{6}\right)
$$

where $p$ and $M$ are expressed in GeV. Lines of equal path length are shown in Fig. 10.

The shortest track that can be detected in a bubble chamber is a few millimeters. Even at the highest Fermilab energies, one is unlikely to identify a charmed particle of mass greater than about $2 \mathrm{GeV}$ via its track in a bubble chamber. On the other hand, emulsions are sensitive to tracks as short as several tens of microns: onehundredth the length detectable in a bubble chamber. IIsing emulsions one thus can hope to see charmed particles whose mass is less than about $4 \mathrm{GeV}$.

Several suggestive signatures could identify a charmed particle track.

a) short invisible track leading to $N$-prong " $V$ ", N even, éspecially if $N \geq 4$.

b) shor track decaying to an odd number $\mathrm{N}^{\prime}$, especially if $N^{-} \geq 5$.

\section{c) short track decaying with large transverse momentum}

The major difficulty in an emulsion, even given a sufficiently low-mass charmed particle, might well be the accumulation of sufficient statistics. While charmed particles could be produced in pairs, and hence via the strong interactions, one has no way to estimate the effects of their relatively heavy mass on production, and an estimate by Snow (1973)

$$
\sigma \sim M^{-2}
$$

seems the most optimistic. For a $4 \mathrm{GeV}$ charmed particle, this could suppress charmed particle production by nearly $10^{-3}$ relative to pion production. Consequently, only emulsion experiments with at least 
several thousand events (at Fermilab energies) begin to place useful bounds on charmed particle production.

\section{SUMMARY AND CONCLUSIONS}

We have suggested some phenomena that might be indicative of charmed particles. These include:

a) "direct'lepton production,

b) large numbers of strange particles,

c) narrow peaks in mass spectra of hadrons,

d) apparent strangeness violations,

e) short tracks, indicative of particles with lifetime of order $10^{-13} \mathrm{sec}$.

f) di-lepton production in neutrino reactions,

g) narrow peaks in $\mathrm{e}^{+} \mathrm{e}^{-}$or $\mu^{+} \mu^{-}$mass spectra,

h) transient threshold phenomena in deep inelastic leptoproduction,

i) approach of the $\left(\mathrm{e}^{+} \mathrm{e}^{-} \rightarrow\right.$ hadrons $) /\left(\mathrm{e}^{+} \mathrm{e}^{-} \rightarrow \mu^{+} \mu^{-}\right)$ratio to $3 \frac{1}{3}$,

- perhaps from above, and

j) any other phenomena that may indicate a rinass scale of 2-10 GeV.

Unfortunately, we have not answered the most important question of all: "What would constitute a definitive experiment that would lead us to give up the idea of charm (or sóme new hadronic degree of freedom) altogether?" We have tried to indicate some of the reasons why this question still can't be answered properly.

1) The calculations of the charmed quark masses do not give 
charmed particle masses. To obtain the latter directly one must apparently.resort to questionable pole models or spectral-function approaches. The scale in all these approaches, however, is roughly the same: of the order of a few $\mathrm{GeV}$. One must probably keep an open mind for charmed particles as massive as $10 \mathrm{GeV}$. These are still entirely within the range of present day accelerators.

2) The strong interactions are not well enough understood to estimate the associated production of a pair of very massive particles. One might expect the (charmed particle)/(pion) ratio to increase at high transverse momenta, in analogy with the case for kaons. (The large number of pions at small transverse momenta may well come from decays of resonances, which greatly prefer to decay to pions rather than to kaons.) One should probably be prepared for charmed particle pro-. dúction to be at least as suppressed as baryon-antibaryon pair production, in any given kinematic region.

3) The estimates of total charmed particle lifetime are hampered by our ignorance of factors which may enhance nonleptonic decays. By analogy with known cases one can expect such factors to range from 1 to 100 , with $\sim 20$ a reasonable guess based on the hyperons. It is much easier to estimate leptonic decays, since the basic weak interaction of the charmed quark is specified. Even here, however, the qualitative conclusions differ widely depending on whether the charmed particle mass is 2 or $10 \mathrm{GeV}$. In the former case a few channels are important, while in the latter some kind of "inclusive" est imate is needed.

We have estimated, for the semileptonic decays

$$
\Gamma_{S_{L}} \simeq 10^{i 1} \sec ^{-1}[M(\mathrm{GeV})]^{5}
$$

and, for the total decay rate,

$$
\Gamma_{\text {iot }}=10^{12} \sec ^{-1}[M(C, v)]^{5}
$$

Certain charmed particles may not have enhanced nonleptonic decays if their final states are "exotic." The semileptonic decays of such states might compete more favorably with the nonleptonic ones, and such particles (the $D^{ \pm}$) are the ones for which tracks in emulsions are most likely to be found.

Many of the tests suggested above would not even be conclusive evidence for charmed particles if their results were positive. No test is conclusive which does not lead to a measurement of the charmed particle mass. For example, since all semileptonic decays of charmed particles to uncharmed ones involve $|\Delta Q|=1$; direct lepton production cannot be, invoked by itself as evidence for charm, since (because of the missing neutrino) a mass measurement will not be possible.

The most convincing evidence for charmed particles would come from observation of short tracks. These have been looked for in bubble chambers and the results are negative ${ }^{23}$ so far. However, further emulsion searches are desirable.

It is quite likely that charmed particles might live too short a time to make any visible track. In that case, the most conclusive 
evidence for their existence would be the detection of narrow peaks in multi-particle mass spectra. Missing-mass spectra are generally not adequate since the charmed particles are produced in pairs or via neutrinos (which do not lend themselves to missing-mass studies). Consequently; high resolution; high-statistics effective-mass multiparticle spectrometers hold the best promise for detection of charmed particles if they are too short-lived to make visible tracks.

One can only hope that we shall be rescued from the problem of charm either by experimentalists -- who find it--or by ingenious theorists-who show us how to do without it while still accounting for the remarkable existence of neutral $\Delta S=0$ currents.

Finally, we ask, "Could any charmed particles have been seen?" There are a few candidates, and we shall discuss them briefly.

- (1) In the experiment of Christenson et al.(1970) at AGS, the reaction

$$
p+U \rightarrow \mu^{+}+\mu^{-}+\text {ariything }
$$

was studied. The differential cross section in the effective mass of the muon pair was found to have a "shoulder" in the mass region near $3.5 \mathrm{GeV} / \mathrm{c}^{2}$. The authors commented that the observed spectrum could be reproduced as a composite of a narrow.vector boson resonance and a steep continuum when the single-particle mass resolution and efficiency were properly introduced into their analysis. Could this phenomenon be due to the production of $\phi_{\mathrm{c}}$ which decays copiously into muon pairs? If it is, the production cross section of $\phi_{c}^{\prime}$ is about $10^{-32} \mathrm{~cm}^{2}$ at $p \simeq 30 \mathrm{GeV} / \mathrm{c}$, assuming the branching ratio of a few percent into the muon pair channel.

(2) Niu, et al.(1971) reported on a cosmic ray event in which a heavy particle decayed into a charged particle and a neutral, which subsequently decayed into $2 y$. They associate the $2 y$ with $\pi^{\circ}$ decay. The $2 y$ carried the energy of $3.2 \pm 0.4 \mathrm{TeV}$, and the charged particle $0.59 \mathrm{TeV}$. The transverse momentum carried by the decay particles with respect to the flight direction of the parent was $(627 \pm 90) \mathrm{MeV} / \mathrm{C}$. It is tempting to speculate that this event was a two-body decay of a charmed particle, for example, $F^{ \pm} \rightarrow \pi^{ \pm} \eta \rightarrow \pi^{ \pm} \gamma \gamma$. If this is correct, then the mass of the parent is about $2 \mathrm{GeV} / \mathrm{c}^{2}$, and its lifetime about $10^{-11} \mathrm{sec}$

(3) Di-muon events reported by the Harvärd-PennsylvaniaWisconsin collaboration (Rubbia, 19.74) at Fermilab could be due to the $v$-production of charmed particles which decay leptonically. 
-72- FERMILAB-Pub-74/86-THY

\section{ACKNOWLEDGMENTS}

We have enjoyed many beneficial discussions with our colleagues. Particularly we wish to thank S. L. Adler, R. Dashen, T. Ferbel, J. -M. Gaillard, L. Lederman, H. Lipkin, A. Pais, E. Paschos, J. Prentki, C. Quigg, G. Snow, S. B. Treiman, S. Weinberg and

J. Whitmore, as well as the many experimenters to whose questions we have addressed our attention.
.

REFERENCES

Adler, S. L., 1974, to be published.

Alper, B., et al. , 1973, Phys. Lett. 44B, 521.

Altarelli, G. and Maiani, L., 1974, Rome preprint.

Altarelli, G. , Cabibbo, N., and Maiani,' L., 1974, Phys. Lett. B48, 435.

Amati, D., Bacry, H., Nuyts, J., and Prentki, J.', 1964a, Phys. Lett.

11, 190.

Amati, D., Bacry, H., Nuyts, J., and Prentki, J., 1964b, Nuovo Cimento 34,1732 .

Appel, J.A., Bourquin, M. H., Gaines, I. , Hom, D. C., Lederman,

L. M. , Paar, H. P., Repellin, J.-P., Saxon, D. H. , Snyder,

... W. D., Weiss, J.M., Ỵoh, J.K., Brown, B.C., Gaillard, J.-M. and Yamanouchi, T., 1974a, Phys. Rev. Lett. 33, 719.

Appel, J.A., et al., 1974b, Phys. Rev. Lett. 33, 722.

Appelquist, T. and.Georgi, H., 1973, Phys. Rev. D8, 4000.

Aubert, B., et al., 1974, Phys. Rev. Lett. 32, 1454. -

Banner, M. , et al. , 1973, Phys. Lett. 44B, 537.

Bardeen, W. A., Drell, S. D., Weinstein, M. and Yan, T. M., 1974,

to be published.

Barish, B. C. ,et al. , 1974a, paper No. 588, XVII International

Conference on High Energy Physics, London.

Barish, S. J. et al., 1974b, Phys. Rev. Lett. 33, 448.

Bars, I. , Halpern, M. B., and Yoshimura, M., 1972, Phys. Rev. Lett. 29. 969 
Bars, I., Halpern, M. B., and Yoshimura, M., 1973, Phys. Rev. D7, 1233.

Benvenuti, A., et al., 1974, Phys. Rev. Lett. 32, 800.

Berkelman, K. .. 1971, Proceedings of the 1971 International Symposium

on Electron and Photon Interactions at High Energies, 263 ,

(Cornell University, Ithaca, N. Y.).

Bjorken, B. J. and Glashow, S. L., 1964, Phys. Lett. 11, 255.

Bouchiat, C., Iliopoulos, J., and Meyer, P. H., 1972, Phys. Lett.

38B, 519.

Boymond, J. P., et al., 1974, Phys. Rev. Lett. 33, 112.

Büsser, F. W., et al., 1973, Phys. Lett. 46B, 471.

Cable, G. D. .. Hildebrand, R. A., Pang, C.Y., and Stiening, R.,

1974, Phys. Rev. D (to be published).

Carey, D.C., Johnson, J.R., Kammerud, R., Peters, M., Ritchie,

D. J., Roberts, A., Sauer, J. R., Shafer, R., Theriot, D.,

Walker, J: K., and Tayler, F.E., 1974, Fermilab preprints

NAL-Pub-74/48-EXP and NAL-Pub-74/49-EXP.

Carlson, C. E. and Freund, P. G. O., 1972, Phys. Lett. 39 B, 349.

Chodos, A., Jaffe, R. L., Johnson, K., Thorn,. C. B. and Weisskopf,'

V.F., 1974, MIT preprint MIT-CंTP-387-Rev.

$-75-$

FERMILAB-Pub-74/86-THY

Christenson, J.H., Hicks, G. S., Lederman, L. M., Limon, P. J., Pope, B. G., and Zavattini, E., 1970, Phys. Rev. Lett. 25, 1523.

Cronin, J. W., Frisch, H. J., Shochet, M. J., Boymond, J. P. ,

Mermod, R., Piroué, P.A'. and Sumner, R. L., 1973, Phys. Rev. Lett. 31, 1426.

DeRujula, A., Georgi, H., Glashow, S. L., and Quinn. H. R., 1974. Rev. Mod. Phys., 46, 391.

de Wit, B., 1973, Nuci. Phys. B57, 237.

Dittner, P., Eliezer, S. and Kuo, T.K., 1973, Phys. Rev. Lett. 30 . 1274 .

Eliezer, S., 1974, Imperial College preprint.

Farrar, G. , 1974, Preprint CALT-68-422.

Gaillard, M. K. . 1974, in Proceedings of the IV International

Conference on Neutrino Physics and Astrophysics, Philadelphia.

Gaitlard, M. K. and Lee, B. W. , 1974a, Phys. Rev. D (to be published August 1, 1974).

Gailiard, M. K. and Lee, B. W., 1974b, Phys. Rev. Lett. 33, 108. Gavrielides,..A., 1974, thesis, University of Minnesota (unpublished). Gell-Mann, M. and Leutwyler, H., 1973, Caltech preprint CALT-68409.

Gell-Mann, M., Oakes, R. J., and Renner, B., 1968, Phys. Rev. 175, 2195. 
Georgi, H. and Glashow, S. L., 1974, Phys. Rev. Lett. 32, 438. Glashow, S. L. and Weinberg, S, , 1968, Phys. Rev. Lett. 20, 244. Glashow, S. L. , Iliopoulos, J. and Maiani, L., 1970, Phys. Rev. D2, 1285.

Glashow, S. L. , 1974, Proceedings of the IX International Conference on Experimental Meson Spectroscopy. Boston.

Gottfried. K., 1971. in Proceedings of the 1971 International Symposium on Electron and Photon Interactions at High Energies, 221 (Cornell Univer sity. Ithaca, N.Y.).

Hara, Y., 1964, Phys. Rev. 134, B701.

Hasert, F. et al., 1973, Phys. Rev. Lett. 46B. 121 and 138.

Hayes. S. Imilay, R., Joseph. P. M. . Keizer; A. S, Knowles, J., and Stein, F.C., 1970, . Phys. Rèv. Lett. 24, 1369.

Joglekar, S., 1974, to be published.

Langacker, P. and Pagels, H., 1973, Rockefeller University preprint COO-2232B-32A.

Lee, B. W. , 1972, "Perspectives in the Theory of Weak Interactions," in Proceedings of, the XVI International Conference on High Energy Physics, (NAL) Vol. IV.
Lee, W: et al. , 1974, XVII International Conference on High Energy Physics, London.

Lipkin, H., 1965a, in Proceedings of the Second Coral Gables Conference, Symmetry Principles at High Energies, 202 (W. H. Freeman and Co., San Francisco).

Lipkin, .H., 1965b, "Lie Groups for Pedestrians", second Edition. (North Holland Publishing Co..., Amsterdam).

Litke, A., et a1. , 1973, Phys. Rev. Lett., 30, 1189.

Llewellyn-Smith, C. H., 1972, Phys. Reports 3C, 261.

Ma, E., 1974, Phys. Rev. D. 3103.

Maki, Z., and Ohnuki, Y., 1964, Prog. Theor. Phys. (Kyoto), 32, 144.

Niu, K., Mikumo, E., and Maeda, Y., 1971, Prog. Theor. Phys. 46 1644 .

Pati, J. C. and Salam, A., 1974, Phys. Rev. D10, 275.

Richter, B., 1974, in Proceedings of the XVII International Conference on High Energy Physics in London, England, to be published.

Rubbia, C., 1974, in Proceedings of the XVII International Conference on High Energy Physics, London.

Salam, A., 1968, Proceedings of the 8th Nobel Symposium (Amquist and Wiksell, Stockholm). 
Snow, G., 1973, Nucl. Phys., and Electromagnetic and Weak Interactions (8th Rencontre de Morcond), 379 (CNRS).

Tarnopolsky, G., et al., 1974, Phys. Rev. Lett. 32, 432.

Vainshtein, H. I. and Khriplovich, I. B., 1973, ZhETF Pis. Red. 18,

141

Weinberg, S., 1967, Phys. Rev. Lett. 19, 1264.

Weinberg, S., 1971, Phys. Rev. Lett. 28, 1688.

Weinberg, S., 1973, Phys. Rev. D8, 605.

Weinberg, S., 1974a, "Recent Progress in' Gaugè Theories of the

Weak, Electromagnetic and Strong Interactions, "Rev. Mod.

Phys., 46, 255.

Weinberg, S. , 1974b, in Proceedings of the XVII International

Conference on High Energy Physics, London, to be published. Zee, A., 1973, Phys. Rev. D8, 4038.

\section{FOOTNOTES}

${ }^{1}$ For reviews see Lee, 1972; and Weinberg., 1974a.

${ }^{2}$ Hasert, et al., 1973; Benvenuti, et al., 1974; Aubert, et al., 1974;

Barish, et al., 1974a, b; and Lee, et al., 1974.

${ }^{3}$ Amati, et al. , 1964a; Bjorken and Glashow, 1964; Maki and Ohnuki,

1964; Hara, 1964; Glashow, et al., 1970; Weinberg, 1971; and Bouchiat. et al., 197.2 .

${ }^{4}$ Weinberg, 1967; Salam, 1968.

${ }^{5}$ Bars, et al. , 1972, 1973; deWit, 1973.

6. Weinberg, 1973, 1974a.

${ }^{7}$ Gaillard and Lee, 1974a; Jainshtein and Khriplovich, 1973; Ma, 1974; Gavrielides, 1974.

${ }^{8}$ Charmed particle searches have previously been discussed by Carlson and Freund, 1972; Snow, 1973; and Glashow, 1974.

${ }^{9}$ Construction of SU(4) representations has been discussed in detail by Amati, et al., 1964b and also by Lipkin, 1965a,b.

${ }^{10} \mathrm{~A}$ possible resolution has been suggested by Langacker and Pagels, 1973.

${ }^{11}$ Chodos, et al. , 1974; Gell-Mann and Leutwyler, 1973; Bardeen, et al. , 1974.

${ }^{12}$ In fact, the operator which is enhanced by the mechanism discussed 
by Gaillard and Lee, 1974b, does not contribute to the decay $D^{ \pm} \rightarrow \mathrm{K} \pi$ in the SU(3) limit. However, 3-body decays of $D^{ \pm}$are not forbidden and current algebra arguments indicate that they should be comparable in strength to $\mathrm{D}^{0} \rightarrow \mathrm{K} 2 \pi$.

${ }^{13}$ In $F^{*} \rightarrow F \gamma$, only the isoscalar current contributes: Assuming the coupling is comparable to that for $\phi \rightarrow \eta \gamma$, we obtain

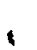

$$
\begin{aligned}
\Gamma\left(F^{*} \rightarrow F y\right) & \leqslant\left(\frac{m_{\phi}}{m_{F}^{*}}\right)^{3}\left(\frac{m_{F *}^{2}-m_{F}^{2}}{m_{\phi}^{2}-m_{\eta}^{2}}\right)^{3} \Gamma(\phi \rightarrow \eta y) \\
& \simeq\left(\frac{G e V}{m_{F}^{*}}\right)^{3} \times 10^{20} \mathrm{sec}^{-1}
\end{aligned}
$$

where we have used the mass relations $(3.6),(3.8)$ and $(4.15)$.

${ }^{14}$ H. Lipkin, private communication.

15. A. Llor et and P. Musset, private communication.

${ }^{16}$ This parametrization has been suggested by Farrar, 1974 and is consistent with the data (N. Stanko, private communication).

${ }^{17}$ Appelquist and Georgi, 1973; Zee; 1973.

${ }^{18}$ Busser, et al., 1973; Alper, et al., 1973; Banner, et al., 1973.

${ }^{19}$ J. - M. Gaillard, private communication.

${ }^{20}$ This is a modification by J. -M. Gaillard of the parametrization suggested by Carey, et al., 1974.
${ }^{21}$ This is a modification by Appel, et al., 1974a of the CCR fit, given in Eq. (5.24). (See footnote 18).

22 Boymond, et al., 1974; Appel, et al., 1974b. A lower yield $\{\simeq 0.25$ $\times 10^{-4}$ ) was $r$ reported by the Serpukhov group at the XVII International Conference on High Energy Physics, London 1974.

${ }^{23}$ T. Ferbel, private communication. 


\section{FIGURE CAPTIONS}

Weight diagrams for SU(4). Shaded planes denote multiplets of $\mathrm{SU}(3), \mathrm{U}(1)_{\mathrm{C}}$

a) The four quarks of $\mathrm{SU}(4)$ : the conventional $\mathrm{SU}(3)$ triplet consisting of u ("up"), d ("down") and s ("strange") with $C=0$, and an $\operatorname{SU}(3)$ singlet c ("charmed") with $\mathrm{C}=1$,

b) The three-quark $1 / 2^{+}$baryons which form a 20-plet of SU(4). The SU(3) multiplets are $8(C=0), 6+\overline{3}_{2}(C=1)$ and $\underbrace{}_{2}(C=2)$.

c) The 15-plet + singlet pseudoscalars. The SU(3) multiplets are $3_{\sim}(C=-1), \underset{\sim}{8}+1(C=0)$ and $\overline{3}(C=+1)$.

Fig. 2
Schematic representation of charmed pseudoscalar decays. The arrow points from the parent particle to a state with the quantum numbers of the final state hadronic system.

a) Semi-leptonic decays with $\Delta S=\Delta C\left(\Gamma \propto \cos ^{2} \theta_{C}\right)$.

b) Non-leptonic decays with $\Delta S=\Delta C\left(\Gamma \cdot \propto \cos ^{4} \theta_{c}\right)$. .

c) Non-leptonic decays with $\Delta S \not \Delta C \mathbb{C} \propto \cos ^{2} \theta_{C}$ $\left.\sin ^{2} \theta_{c}\right)$
- Schematic representation of strong decays of charmed

baryons. The mass splittings within an SU(3) multiplet are independent of the charm mass scale R. The scale for multiplet separation is linear in $R$ (see Eqs. 3.9).

Fig. 4

.


r:are arbitrarily smoothed over the step function behavior which appears in Eq. (5. 20).

Fig. 8

Data on the ratio $R=\sigma\left(e_{\bar{e}}^{-} \rightarrow\right.$ hadrons $) / \sigma\left(e_{\bar{e}}^{-} \rightarrow \mu \bar{\mu}\right)$, and predictions of the asymptotically free quark model without charm (lower curve) and with charm (upper curve). a) CEA data: Litke, et al., 1973: Tarnopolsky, et al., 1973; b) SLAC-LBL data: Richter, 1974; c) This is taken from Adler, 1974. Monte Carlo calculation of $r$ [see Eq. (5.25)] as a function of $\mathrm{p}_{\perp}$ for the decay $\mathrm{D} \rightarrow \mathrm{K} \ell \nu$. The curves are calculated for leptons observed at $90^{\circ}$ in the production center-of-mass with $\sqrt{\mathrm{s}}=24 \dot{\mathrm{GeV}}$ and for two assumed production distributions, chosen to reproduce the measured slope $\mathrm{e}^{18}$ at $\mathrm{p}_{\perp}=3 \mathrm{GeV}$ : $E \frac{d^{3} \sigma}{d p^{3}} \simeq\left(1+p_{\perp}^{2}\right)^{-5.5}\left(1-\frac{p}{p_{\max }}\right)^{4}$, dashed curves, ${ }^{20}$ $E \frac{d^{3} \sigma}{d p^{3}}=\left(p_{\perp}^{2}+1\right)^{-4.6} \exp \left(-26 p_{\perp} / \sqrt{s}\right)$, solid curves. ${ }^{21}$ The ratio $r$, calculated for both parent and decay lepton at $90^{\circ}$ in the production center of mass, is not invariant under transformation to the target rest frame. However, the observed lepton-pion yield ratio:

$$
\left(\ell / \pi^{\circ}\right)=\mathrm{r} \times \mathrm{B}_{\ell_{3}} \times\left(\mathrm{D} / \pi^{\circ}\right)
$$

is invariant, since both leptons and pions are effectively massless and undergo the same Lorentz transformation. Here $\mathrm{B}_{\ell_{3}}$ is the branching ratio for the $\mathrm{K} \ell \mathrm{v}$ mode of $\mathrm{D}$ decay. Courtesy J. $-\mathrm{M}$. Gaillard. Path length versus mass assuming $T M^{5}=10^{-12} \operatorname{sec~GeV}$. Path length $\ell$ is then

$$
\ell=300 \mu \times\left(\mathrm{p} / \mathrm{M}^{6}\right)
$$

where $\mathrm{p}$ and $\mathrm{M}$ are in $\mathrm{GeV} / \mathrm{c}, \mathrm{GeV} / \mathrm{c}^{2}$, respectively. 

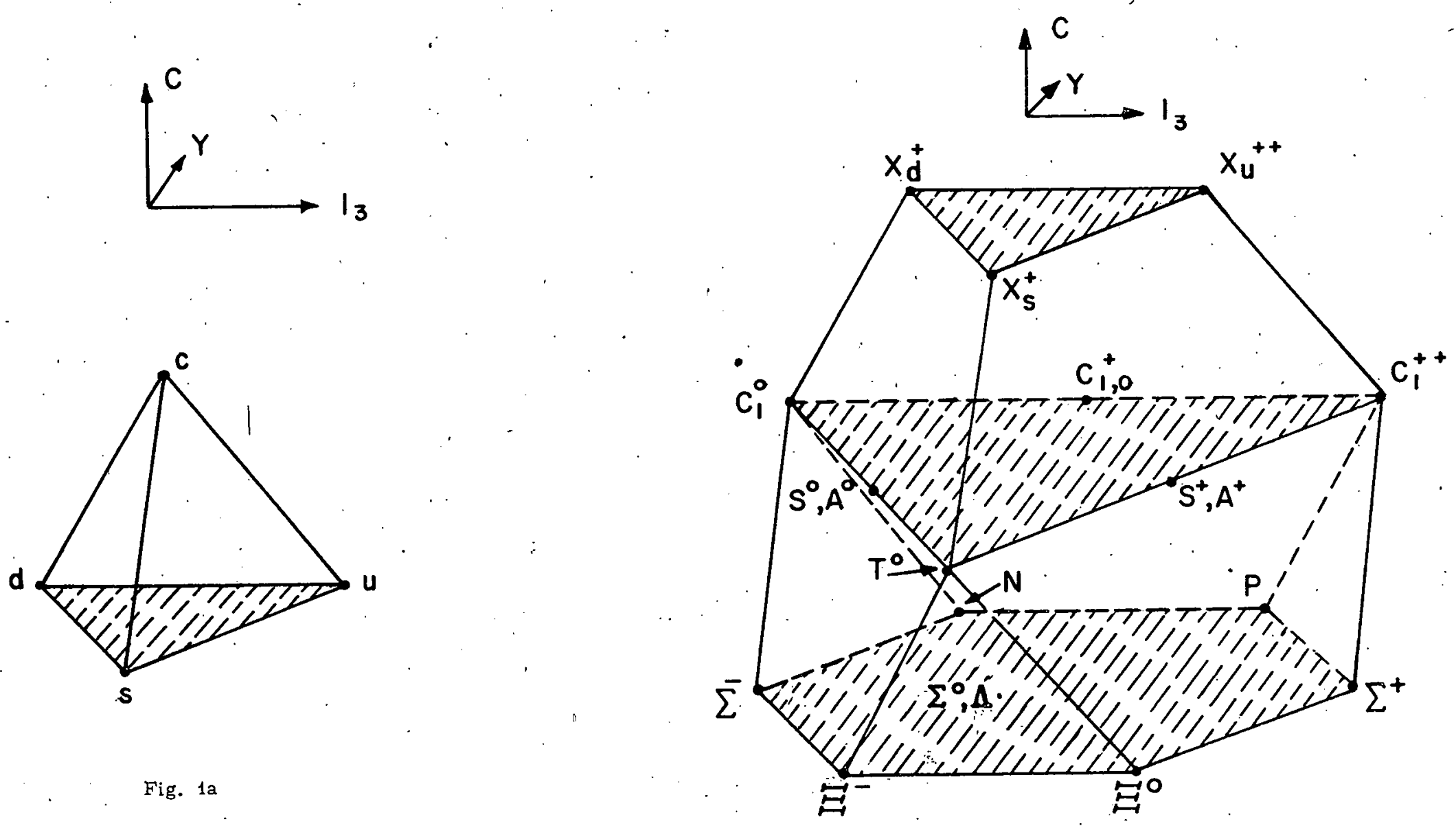

Fig.' $1 b$ 

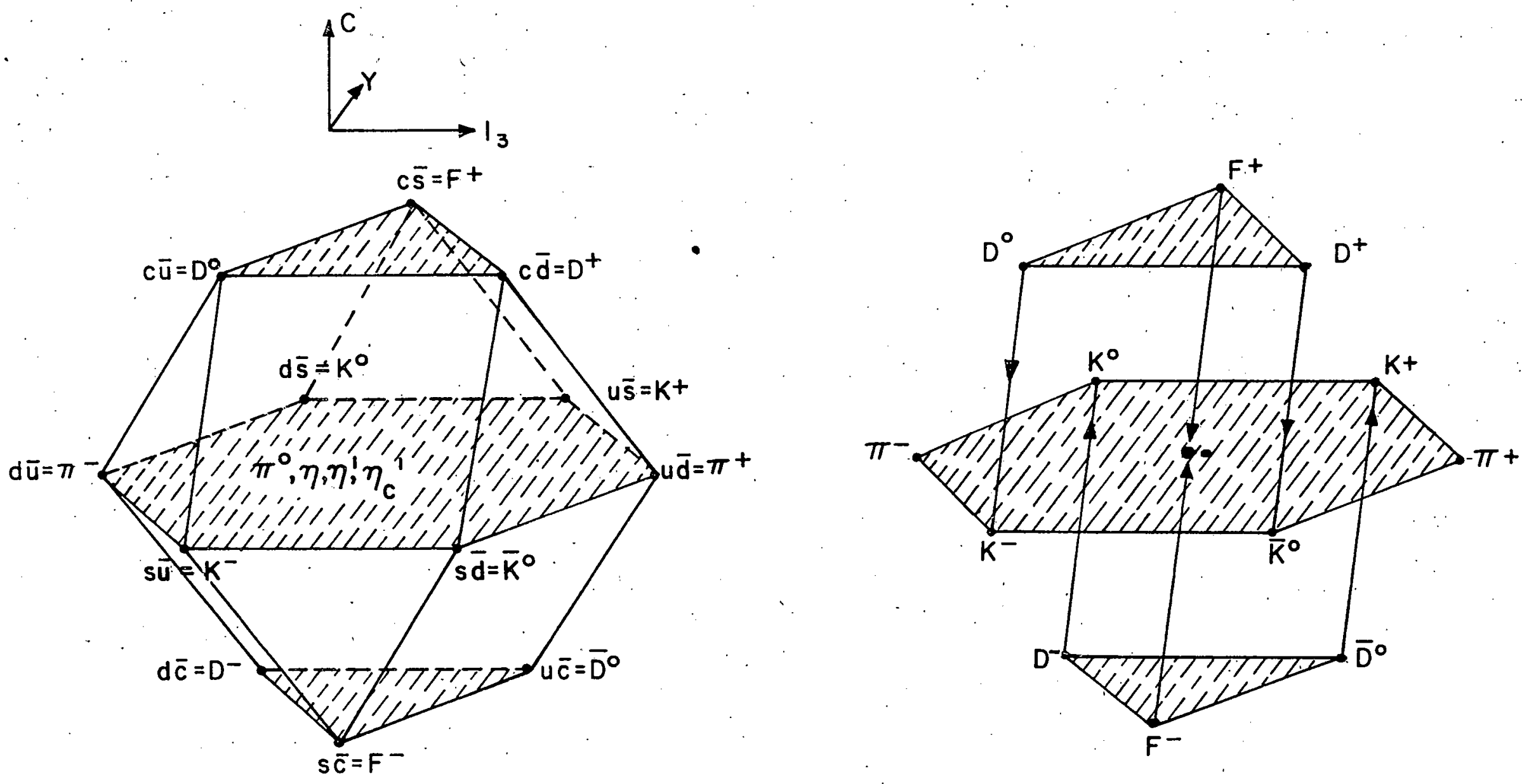

Fig. 2a

Fig. 1c 


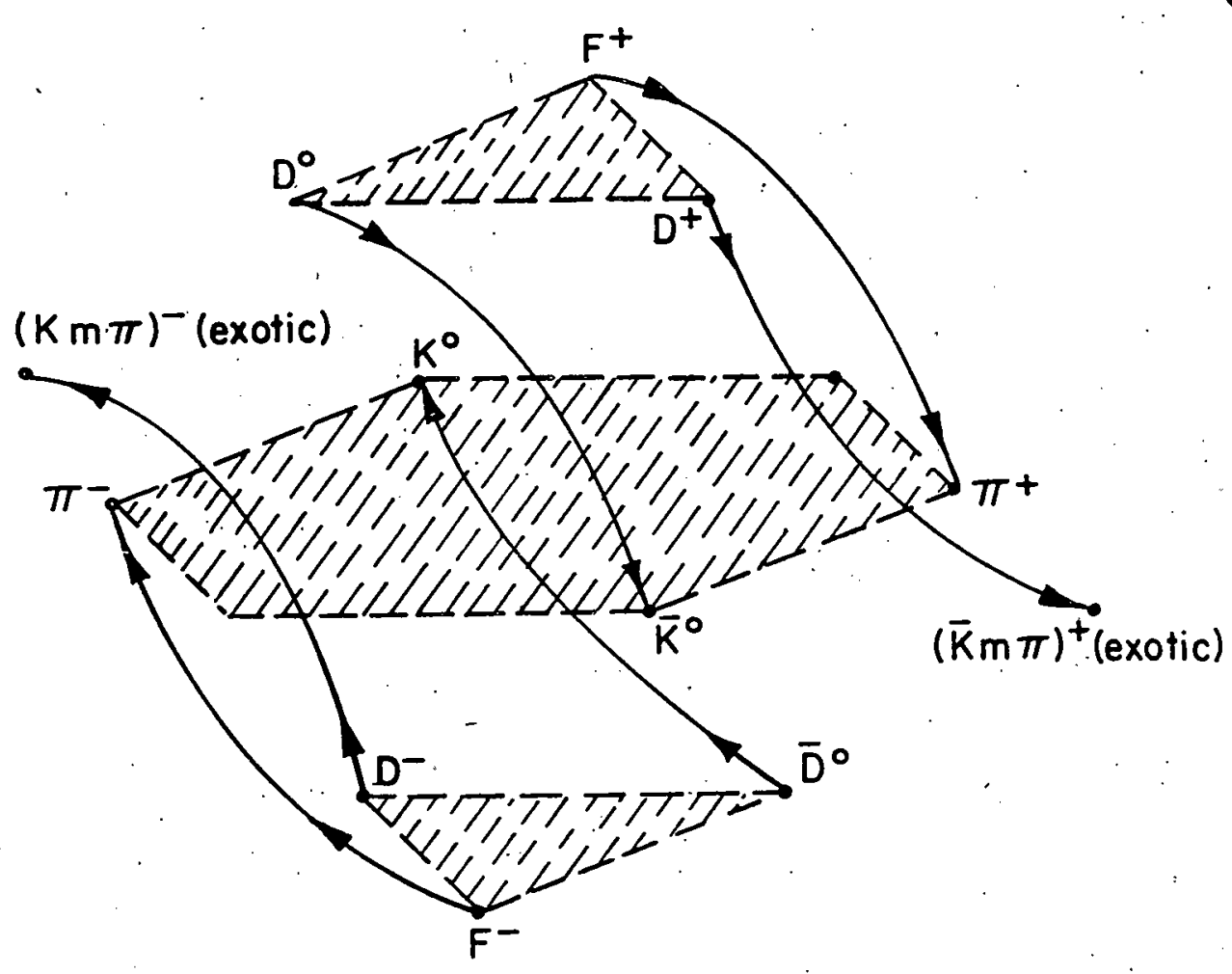

Fig. 2b

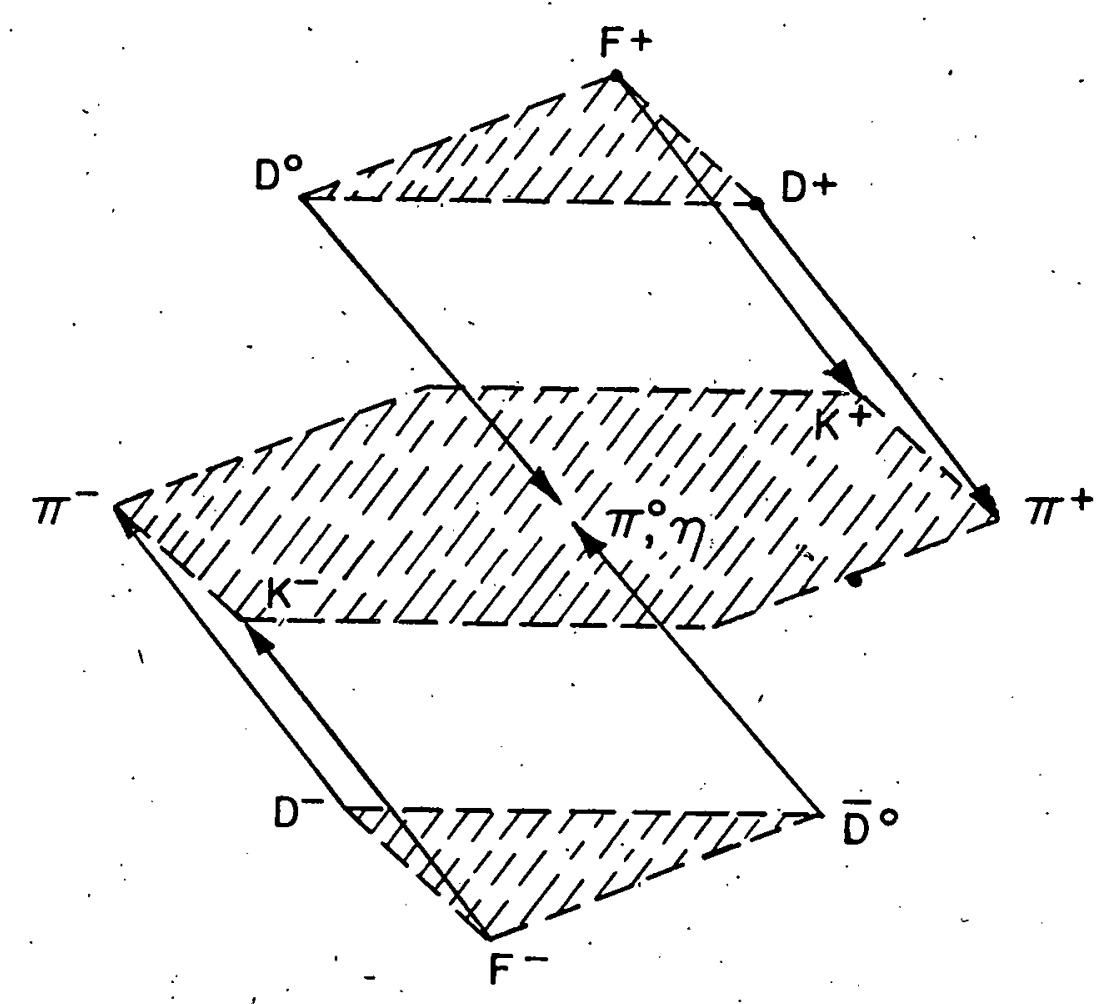

Fig. 2c 

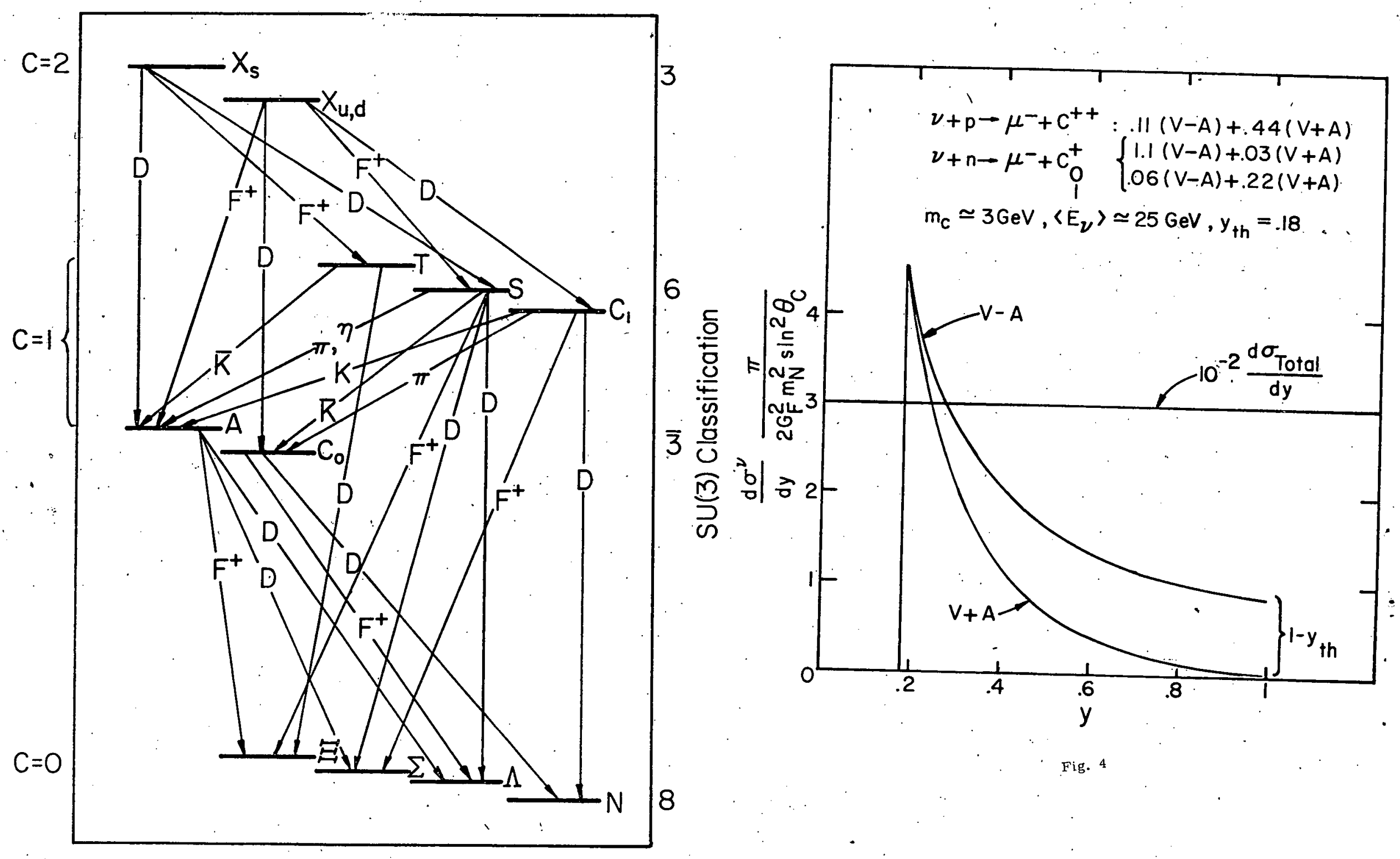


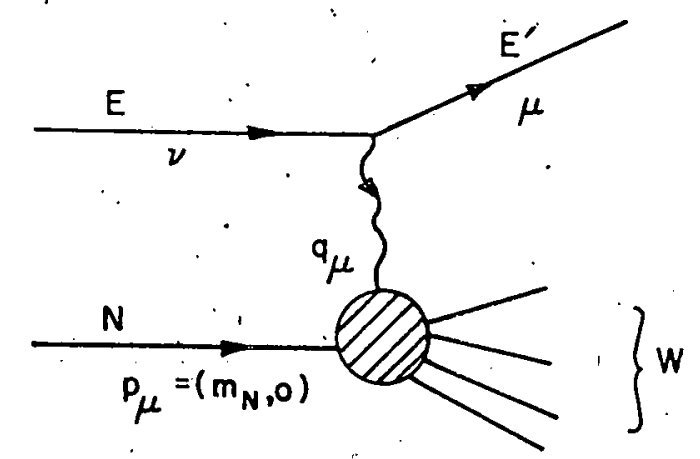

Fig. 5

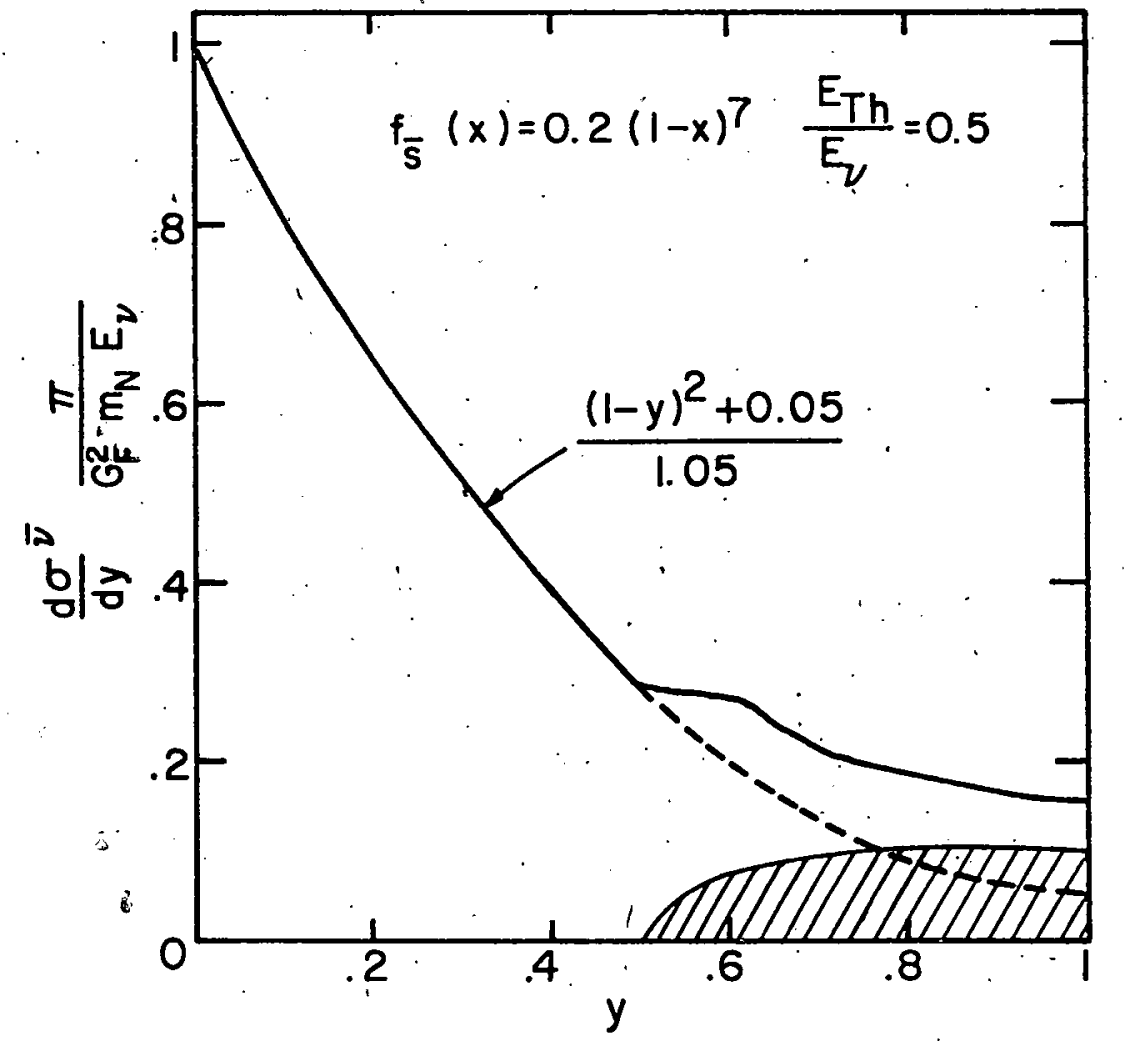

Fig. 6 

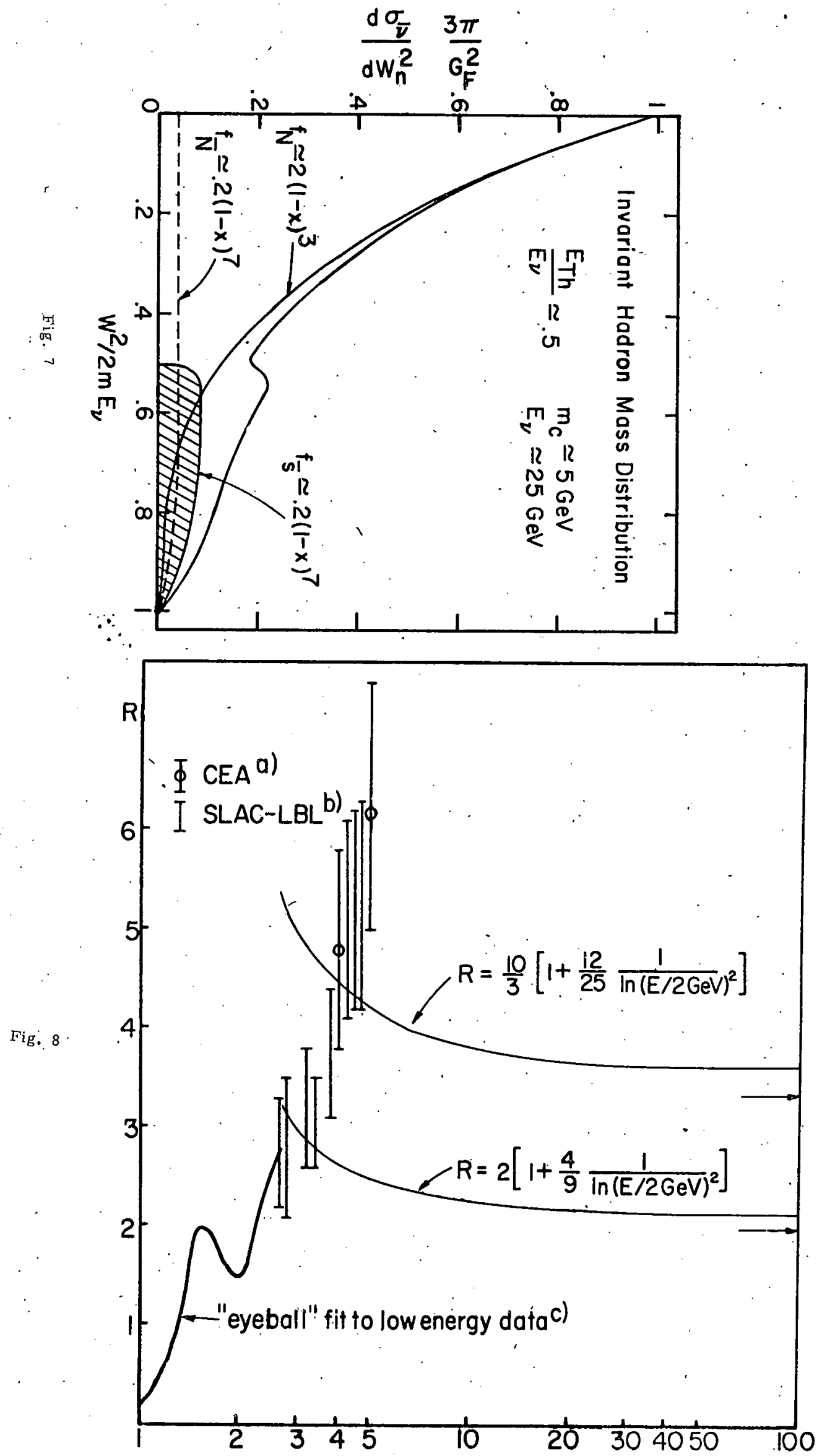


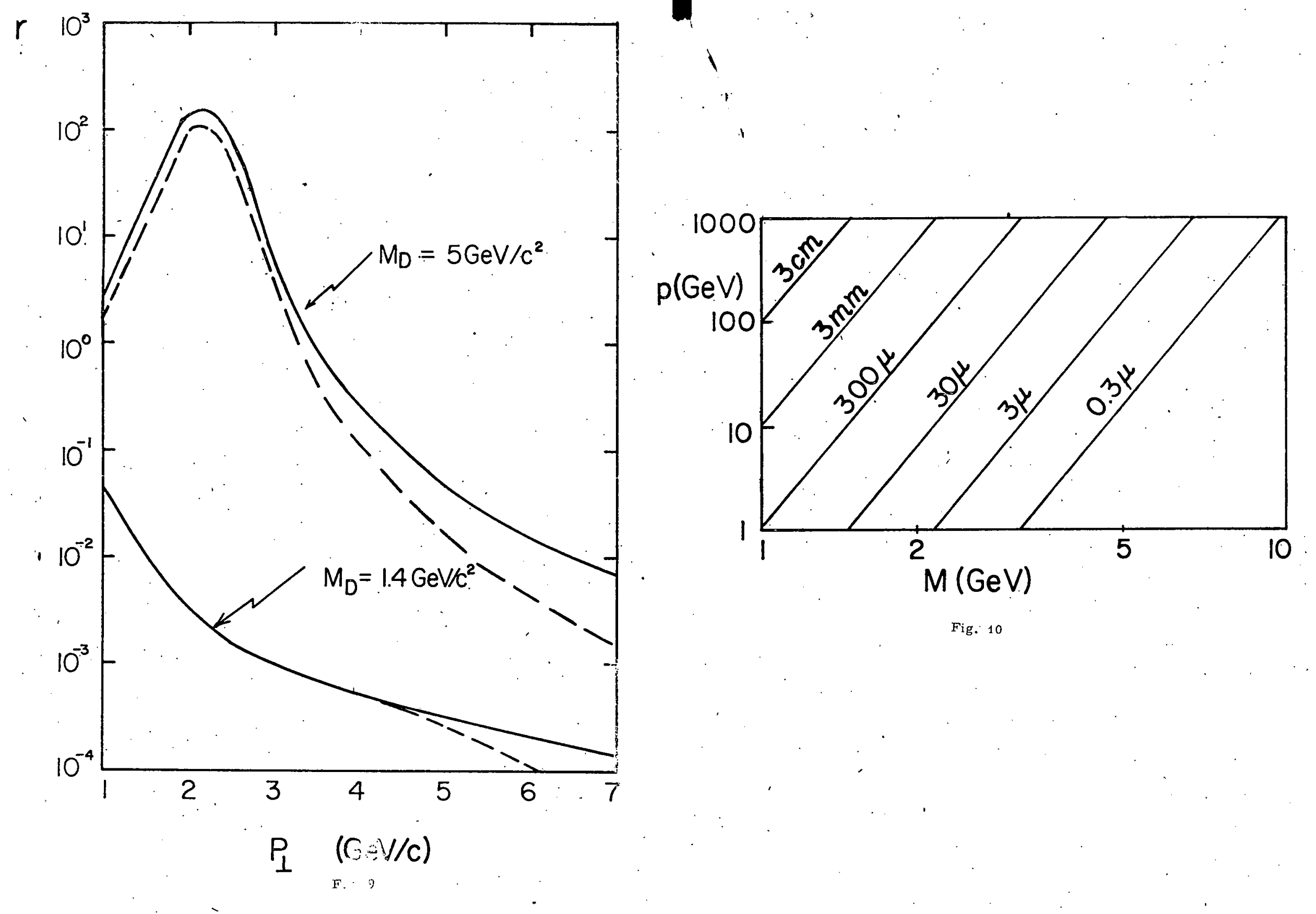

